\title{
Parallel Preconditioners for Monolithic Solution of Shear Bands
}

\author{
Luc Berger-Vergiat, Colin McAuliffe, Haim Waisman* \\ Department of Civil Engineering \& Engineering Mechanics, Columbia University, \\ New York, NY 10027
}

\begin{abstract}
Shear bands are one of the most fascinating instabilities in metals that occur under high strain rates. They describe narrow regions, on the order of micron scales, where plastic deformations and significant heating is localized that eventually leads to fracture nucleation and failure of the component.

In this work shear bands are described by a set of four strongly coupled thermomechanical equations discretized by a mixed finite element formulation. A thermoviscoplastic flow rule is used to model the inelastic constitutive law and finite thermal conductivity is prescribed which gives rise to an inherent physical length scale, governed by competition of shear heating and thermal diffusion. The residual equations are solved monolithically by a Newton type method at every time step and has been shown to yield mesh insensitive result. The Jacobian of the system is sparse and has a nonsymmetric block structure that varies with the different stages of shear bands formation.

The aim of the current work is to develop robust parallel preconditioners to GMRES in order to solve the resulting Jacobian systems efficiently. The main idea is to design Schur complements tailored to the specific block structure of the system and that account for the varying stages of shear bands.

We develop multipurpose preconditioners that apply to standard irreducible discretizations as well as our recent work on isogeometric discretizations of shear bands.

The proposed preconditioners are tested on benchmark examples and compared to standard state of practice solvers such as GMRES/ILU and LU direct
\end{abstract}

\footnotetext{
*Corresponding author

Email address: waisman@civil .columbia.edu (Haim Waisman)
} 
solvers. Nonlinear and linear iterations counts as well as CPU times and computational speedups are reported and it is shown that the proposed preconditioners are robust, efficient and outperform traditional state of the art solvers.

Keywords: Shear bands, Preconditioner, Multiphysics, Schur complement, Schwarz method, GMRES, parallel scalability

\section{Introduction and problem statement}

Shearbanding is the localization of plastic strain into narrow bands that is typically observed in metals (and other materials) undergoing high strain rate loading. Shear bands are characterized by small zones of intense plastic deformations due to thermal softening that results in locally reduced stress bearing capacity of the material and eventually lead to its failure. Modeling shear bands accurately is very challenging due to the complicated multiphysics and difficult numerics. This is a highly nonlinear, coupled thermo-mechanical problem with localized cracklike domains that require specialized discretizations and significant computational power to resolve shear bands accurately in space and time.

Experimentally derived material models for these loading regimes describe plastic flow as being dependent on temperature, strain rate, and a hardening parameter [1]. While several models are available, all are similar in that increasing temperature (due to plastic work) has a softening effect, causing plastic flow to occur more readily, while increases in strain rate and the hardening parameter have a hardening effect.

The experimental work of [2] shows that shear bands form in three stages. In stage 1 the material deforms homogeneously: elastically and plastically and the temperature rises uniformly. Stage 2 marks the onset of shear band localization with a localized temperature rise starting to dominate strain and strain rate harding leading to strain softening effect. Finally, in stage 3, severe localization and rapid softening occurs, so called a stress-collapse phenomena, which indicates a sudden and large drop in the material's load bearing capability. For more discussion on the physics of shear bands, the reader is referred to the reviews in $[3,4,5]$.

Shear bands can be modeled by a set of four nonlinear partial differential equations (PDEs) [1, 6, 7], two of which are balance equations: the conservation of momentum and thermal energy, and two constitutive equations: the elastic and inelastic constitutive relations. Assuming small deformations, these four coupled 
equations, written in tensorial notation, are

$$
\left\{\begin{array}{rll}
\rho \underline{u} & =\underline{\nabla} \cdot \underline{\sigma} \\
\rho c \dot{\bar{T}} & =\kappa \underline{\nabla} \cdot(\underline{\nabla T})+\chi \bar{\sigma} g\left(\bar{\sigma}, T, \bar{\gamma}_{p}\right) & \\
\dot{\underline{\sigma}} & =\underline{C}^{\text {elas }}:\left(\underline{\nabla}^{s} \underline{\dot{u}}-\frac{3}{2} \frac{g\left(\bar{\sigma}, T, \bar{\gamma}_{p}\right)}{\bar{\sigma}} \underline{\underline{S}}-\alpha \dot{T} \underline{I}\right) & \text { in } \Omega \\
\dot{\overline{\gamma_{p}}} & =\overline{\bar{g}}\left(\bar{\sigma}, T, \bar{\gamma}_{p}\right) &
\end{array}\right.
$$

where one, two and four underlines indicate first-, second- and fourth- order tensors respectively; a colon (double dot) indicates the contraction (inner product) operator; an overline indicates an equivalent quantity; $\underline{\nabla}$ and $\underline{\nabla}$. are the gradient and the divergence operators and $\underline{\nabla}^{s}$ is the symmetric part of the gradient operator $\underline{\nabla}^{s}=\frac{1}{2}\left(\underline{\nabla}+\underline{\nabla}^{T}\right)$. The four unknown variables are the displacement field $\underline{u}$, the temperature field $T$, the stress field $\underline{\underline{\sigma}}$ and the equivalent plastic strain (EQPS) field $\bar{\gamma}_{p} . \underline{\underline{S}}$ is the deviatoric part of $\underline{\underline{\sigma}}$ defined as $\underline{\underline{S}}=\underline{\underline{\sigma}}-\frac{\operatorname{tr} \underline{\underline{\sigma}})}{3} \underline{\underline{I}}$ and $\bar{\sigma}$ is the Von Mises equivalent stress defined as $\bar{\sigma}=\sqrt{\frac{3}{2} \underline{\underline{S}}: \underline{\underline{S}}}$ (see [8]); and $\underline{\underline{I}}$ is the second order identity tensor. The material parameters in the model are: $\rho$ the material density, $c$ the specific heat, $\kappa$ the thermal conductivity, $\chi$ the Taylor-Quinney coefficient which defines the amount of plastic work converted to heat, $\alpha$ the thermal expansion coefficient, $\underline{\equiv}^{\text {elas }}$ the elastic forth order tensor and the flow law. The plastic flow law used is a modified Litonski law [9] that has been used in the literature for shear band modeling $[10,11]$. This flow law has the particularity of not introducing an explicit yield limit. Arguably the use of a plastic law with an explicit yield limit would lead to more nonlinear iterations [12] but this is ultimately not computationally intensive since it will only affect one or two time steps and can be resolved by adaptive time stepping. The expression of the flow law used is provided below

$$
g\left(\bar{\sigma}, T, \bar{\gamma}_{p}\right)=\dot{\bar{\gamma}}_{p, r e f}\left[\frac{\bar{\sigma}}{\sigma_{r e f}\left\{1+\bar{\gamma}_{p} / \bar{\gamma}_{p, r e f}\right\}^{N}\left\{1+\delta-\delta \exp \left(\frac{T-T_{r e f}}{k}\right)\right\}}\right]^{m} .
$$

The four parameters $T_{r e f}, \sigma_{r e f}, \bar{\gamma}_{p, r e f}$ and $\dot{\bar{\gamma}}_{p, r e f}$ are the reference temperature, the yield stress and strain and the reference strain rate. $N$ and $m$ are the strain hardening and rate sensitivity exponents and $\delta$ and $k$ are thermal softening parameters. $\Omega$ is the problem domain and its boundary $\Gamma=\Gamma_{d} \cup \Gamma_{n}$ and $\Gamma_{n} \cap \Gamma_{d}=\varnothing$, where $\Gamma_{d}$ and $\Gamma_{n}$ are the part of the boundary where Dirichlet and Neumann conditions 
are prescribed, respectively. The boundary conditions applied to the problem are as follow:

$$
\begin{array}{ll}
\underline{u}(\underline{x}, t)=\bar{u}(\underline{x}, t) & \text { on } \Gamma_{d}^{u}, \\
\underline{n} \cdot \underline{\sigma}(\underline{x}, t)=\bar{t}(\underline{x}, t) & \text { on } \Gamma_{n}^{u}, \\
T \overline{(x}, t)=\bar{T}(\underline{x}, t) & \text { on } \Gamma_{d}^{T}, \\
\underline{n} \cdot \underline{q}(\underline{x}, t)=\bar{q}(\underline{x}, t) & \text { on } \Gamma_{n}^{T} .
\end{array}
$$

with the initial conditions

$$
\begin{aligned}
& \underline{u}(\underline{x}, 0)=\underline{u}_{0}(\underline{x}) \\
& \underline{\underline{\sigma}}(\underline{x}, 0)=\underline{\sigma}_{0}(\underline{x}) \quad \text { in } \Omega \\
& \bar{T}(\underline{x}, 0)=\bar{T}_{0}(\underline{x}) \\
& \bar{\gamma}_{p}(\underline{x}, 0)=\bar{\gamma}_{p, 0}(\underline{x})
\end{aligned}
$$

where $\underline{u}_{0}(\underline{x}), \underline{\underline{\sigma}}_{0}(\underline{x}) T_{0}(\underline{x})$ and $\bar{\gamma}_{p, 0}(\underline{x})$ are the initial displacements, stresses, temperature and equivalent plastic strain.

Multiple numerical schemes have been developed to analyze shear bands. The most common approach is to first simplify the governing equations in Eq.(1) by assuming adiabatic conditions in which $\kappa=0$. In other words, since shear band propagation is a fast process while thermal diffusion is a slow one, the later has commonly been neglected in computations $[13,14,15]$. While such an approach simplifies the numerics significantly, it also results in a lack of physical length scale in the system. Hence the system is ill posed and the convergence to a shear band width has been shown to be sensitive to the mesh size $[16,10]$.

To this end, Liu et al. proposed a meshfree method [17, 18] and the multiresolution technique $[19,20]$ to simulate shear bands. In the later the multiresolution technique is regularized by the introduction of three length scales: hardening scale, thermal diffusion scale and a micro-void spacing scale. Other approaches such as viscoplasticity [21], gradient plasticity [22] and thermal diffusion [23] have also been proposed to regularize the shear band problem.

If the adiabatic assumption is lifted and the diffusion term is retained then the governing equations need to be solved simultaneously, which complicates the numerical methods. To keep the cost of the simulation at a low level and include the diffusion term, splitting methods were introduced in [24, 25]. With these methods, the equations in system (1) are solved sequentially without the use of a global nonlinear solver. Nonetheless, when shear banding starts to form in stage 2, errors made by splitting cannot in general be recovered and have the tendency to compound through the simulation leading to solutions that diverge from the experimental observations. More critically these approaches do not always preserve 
the intrinsic length scale of the system which leads to mesh dependent solutions. Other approaches using approximations of the Jacobian matrix such as Picard iterations $[3,5]$ also have similar properties to the splitting methods.

To avoid splitting errors and mesh dependent results simultaneous solution methods, so called monolithic or Implicit Nonlinearly Consistent (INC) schemes are needed. The work by Batra et al. $[3,26,23,27,28]$ and Waisman et al. $[29,30,31]$ shows that concurrent solution of the equations leads to accurate simulation results that are insensitive to mesh size and mesh alignment. However this approach is more expensive as it requires the solution of a nonlinear set of equations. Moreover, due to the multiphysics nature of the problem, the discretized system is typically nonsymmetric which limits the choice of solvers. In addition, one needs to compute the Jacobian of the nonlinear system (1), which can be done numerically using a finite difference approach $[32,33,34,35]$ or analytically, thus reducing some of the numerical burden [29].

The matrix associated with the INC scheme is much larger than that used in splitting methods combined with explicit schemes but typically requires fewer time steps to converge. Therefore, the bottleneck of the monolithic scheme is the linear solver used to solve the update equation.

In order to solve efficiently monolithic schemes, efficient and robust preconditioners for iterative Krylov-type solvers such as GMRES [36] or BICGStab [37] are required. The typical black box algebraic preconditioners such as Jacobi, ILU or algebraic multigrid [38, 39] have difficulties accelerating the solution of (1) due to the highly nonlinear multiphysics present, and the strong coupling of the equations.

Many preconditioners for multiphysics-type problems have been proposed. For example Ipsen [40] proposed a general method based on a Schur complement to precondition nonsymmetric matrices and his work led to the widely used block Schur preconditioners for coupled physics problems [41]. Other block and nonblock preconditioners have been successfully applied to many physical problems such as fluids flow [42, 43, 44], solid mechanics [45, 46], magnetohydrodynamic [47] and mechanics [48].

In solid mechanics problems involving plasticity, the Jacobian varies greatly with the state of the system, and thus even if a preconditioner is effective at one time step, it may not be so at another time step. Therefore a good preconditioner for such a system needs to take advantage of the properties of the physics in order to offer a significant speed up to the Krylov solver.

In this paper two parallel preconditioners to GMRES that take advantage of the 
specific discretization and physics of the problem are proposed and investigated. Both methods are based on a Conservation laws-Constitutive laws partition of the Jacobian and an outer Schur complement method. Then each method approximates the Schur complement term differently. The first approach is based off a multiplicative Schwarz method and is denoted by a Schur-Schwarz preconditioner. The second employs another inner Schur complement and is denoted as Schur-Schur preconditioner. Both preconditioners converge well in serial and parallel and are found to be robust for solving the shear band problem throughout all three stages of the deformation. In contrast, we show that directly preconditioning the Jacobian system or the outer Schur complement by of the shelf methods, such as an ILU(0) method would either diverge or be significantly suboptimal compared to the two proposed preconditioners. The strong and weak scaling of both proposed preconditioners is shown to be better than that of parallel state of practice LU direct solvers. The Schur-Schwarz preconditioner having the best strong scalability and the Schur-Schur the best weak scalability as well as better behavior on higher order isogeometric discretizations.

The paper is organized as follows. In section 2 we present algebraic system of equations which arises from discretization of the governing PDE set in Eq. (1) and the implicit scheme used to solve and advance the system in time. In section 3 we derive the two peconditioners and explain how their particular structure are well adapted to solve system (1). In section 4 we analyze the performance of the proposed preconditioners on benchmark examples. We also implement those on a parallel supercomputer and report the scalability results as compared with existing direct LU solvers. Finally concluding remarks are provided in section 5.

\section{Discrete system and solution algorithm}

The governing equations in system (1) are discretized using mixed element formulations, following the approach described in $[29,30]$. We employ Isogeometric Analysis (IGA) [49, 50, 51] in which NURBS shape functions are used to disceretize the displacement and temperature fields. Furthermore, an irreducible description of the stress and equivalent plastic strain (EQPS) fields is employed, which is similar to a collocation method in which those fields are sampled at the gauss points. This Irreducible-NURBS Shear band Quadrilateral element was introduced in [31] for the accurate solution of shear bands and is referred to as INSQ element. 
In mixed finite element formulation of the system in (1), one defines the unknown variables vector $\mathbf{x}$ and the corresponding residual vector $\mathbf{R}$ as follows

$$
\mathbf{x}=\left[\begin{array}{c}
\mathbf{x}_{u} \\
\mathbf{x}_{T} \\
\mathbf{x}_{\sigma} \\
\mathbf{x}_{\bar{\gamma}_{p}}
\end{array}\right] \quad \mathbf{R}=\left[\begin{array}{c}
\mathbf{R}_{u} \\
\mathbf{R}_{T} \\
\mathbf{R}_{\sigma} \\
\mathbf{R}_{\bar{\gamma}_{p}}
\end{array}\right]
$$

where the lower case letters indicate the corresponding fields.

An implicit Newmark time stepping scheme is used to advance the system in time together with a Newton-Raphson algorithm to solve the resulting nonlinear discrete equations at every time step.

Let the nonlinear residual of the system at time step $i$ be written as $\mathbf{R}\left(\mathbf{x}^{i}\right)=0$, then the updated solution of the problem at the $k^{\text {th }}$ Newton iteration is $\mathbf{x}^{i, k+1}=$ $\mathbf{x}^{i, k}+\delta \mathbf{x}^{i, k}$, where $\delta \mathbf{x}^{i, k}$ is the solution of the following equation

$$
\mathbf{J}\left(\mathbf{x}^{i, k}\right) \boldsymbol{\delta} \mathbf{x}^{i, k}=\mathbf{R}\left(\mathbf{x}^{i, k}\right)
$$

where $\delta \mathbf{x}^{i, k}$ is the solution update vector. It has been shown in [29] that a mixed finite element formulation leads to the following block structure of the Jacobian matrix

$$
\mathbf{J}=\left[\begin{array}{cccc}
\mathbf{J}_{u u} & \mathbf{0} & \mathbf{J}_{u \sigma} & \mathbf{0} \\
\mathbf{0} & \mathbf{J}_{T T} & \mathbf{J}_{T \sigma} & \mathbf{J}_{T \bar{\gamma}_{p}} \\
\mathbf{J}_{\sigma u} & \mathbf{J}_{\sigma T} & \mathbf{J}_{\sigma \sigma} & \mathbf{J}_{\sigma \bar{\gamma}_{p}} \\
\mathbf{0} & \mathbf{J}_{\bar{\gamma}_{p} T} & \mathbf{J}_{\bar{\gamma}_{p} \sigma} & \mathbf{J}_{\bar{\gamma}_{p}} \bar{\gamma}_{p}
\end{array}\right]
$$

where the first subscript of each block indicates which residual equation was differentiated and the second subscript indicates which variable it is differentiated with respect to (e.g. $\mathbf{J}_{u \sigma}=\frac{\partial \mathbf{R}_{u}}{\partial \sigma}$ ). After discretization, the block structure can be further expanded as follow

$$
\mathbf{J}=\left[\begin{array}{cccc}
\mathbf{M}_{u} & \mathbf{0} & \mathbf{K}_{u} & \mathbf{0} \\
\mathbf{0} & \mathbf{M}_{T}+\mathbf{K}_{T}+\mathbf{G}_{T T} & \mathbf{G}_{T \sigma} & \mathbf{G}_{T \bar{\gamma}_{p}} \\
\mathbf{K}_{\sigma} & \mathbf{M}_{\sigma T}+\mathbf{G}_{\sigma T} & \mathbf{M}_{\sigma}+\mathbf{G}_{\sigma \sigma} & \mathbf{G}_{\sigma \bar{\gamma}_{p}} \\
\mathbf{0} & \mathbf{G}_{\bar{\gamma}_{p} T} & \mathbf{G}_{\bar{\gamma}_{p} \sigma} & \mathbf{M}_{\bar{\gamma}_{p}}+\mathbf{G}_{\bar{\gamma}_{p} \bar{\gamma}_{p}}
\end{array}\right]
$$

where $\mathbf{M}$ denotes a mass matrix, $\mathbf{K}$ a stiffness matrix arising from linear material behavior and $\mathbf{G}$ is a matrix corresponding to nonlinear material behaviour which arises from the flow law in Eq. (2). Note that the Jacobian matrix is non symmetric which limits the choice of linear solvers. Furthermore, when the solid is in the 
linear elastic deformation regime, all $\mathbf{G}$ blocks are equal to zero and the structure of $\mathbf{J}$ is simplified to

$$
\mathbf{J}^{\prime}=\mathbf{J}^{l i n}=\left[\begin{array}{cccc}
\mathbf{J}_{u u} & \mathbf{0} & \mathbf{J}_{u \sigma} & \mathbf{0} \\
\mathbf{0} & \mathbf{J}_{T T} & \mathbf{0} & \mathbf{0} \\
\mathbf{J}_{\sigma u} & \mathbf{J}_{\sigma T} & \mathbf{J}_{\sigma \sigma} & \mathbf{0} \\
\mathbf{0} & \mathbf{0} & \mathbf{0} & \mathbf{J}_{\bar{\gamma}_{p} \bar{\gamma}_{p}}
\end{array}\right]=\left[\begin{array}{cccc}
\mathbf{M}_{u} & \mathbf{0} & \mathbf{K}_{u} & \mathbf{0} \\
\mathbf{0} & \mathbf{M}_{T}+\mathbf{K}_{T} & \mathbf{0} & \mathbf{0} \\
\mathbf{K}_{\sigma} & \mathbf{M}_{\sigma T} & \mathbf{M}_{\sigma} & \mathbf{0} \\
\mathbf{0} & \mathbf{0} & \mathbf{0} & \mathbf{M}_{\bar{\gamma}_{p}}
\end{array}\right] .
$$

Up to this point all the properties of the Jacobian matrix described in equations (7)(9) are intrinsic to the mechanical system and completely independent of the discretization used.

Next, we analyze the special properties of the INSQ elements which will be important for development of the preconditioners in Section 3. First, note that the irreducible discretization used for the stress and EQPS fields are spatially decoupled which leads to $\mathbf{J}_{\sigma \sigma}$ and $\mathbf{J}_{\bar{\gamma}_{p} \bar{\gamma}_{p}}$ being block diagonal matrices with block sizes 4 (four stresses) and 1 (one equivalent plastic strain), respectively. Furthermore in the linear elastic regime $\mathbf{J}_{\sigma \sigma}$ is diagonal since the derivatives of the flow law with respect to the variables of the problem are zero in the linear elastic regime. In addition the coupled stress-plasticity term $\mathbf{J}_{\bar{\gamma}_{p} \sigma}$ and the temperature-plasticity term $\mathbf{J}_{\bar{\gamma}_{p} T}$ both vanish. However, note that $\mathbf{J}_{\bar{\gamma}_{p}} \bar{\gamma}_{p}$ remains a diagonal matrix due to the mass term, as shown in Eq. (9). Also note that the temperature-stress term is only one way coupled and hence $\mathbf{J}_{T \sigma}$ vanishes too. The sparsity patterns of the Jacobian matrix in the linear elastic and plastic regimes are illustrated in Figure 1.

In irreducible formulations, it is more convenient for notational purposes to re-group the irreducible blocks (stress and plastic strain) under one augmented matrix $\mathbf{J}_{\eta \eta}$ as follows

$$
\mathbf{J}_{\eta \eta}=\left[\begin{array}{ll}
\mathbf{J}_{\sigma \sigma} & \mathbf{J}_{\sigma \bar{\gamma}_{p}} \\
\mathbf{J}_{\bar{\gamma}_{p} \sigma} & \mathbf{J}_{\bar{\gamma}_{p} \bar{\gamma}_{p}}
\end{array}\right]
$$

Plugging this expression into equation (7) leads to the following more condensed expression for the Jacobian matrix

$$
\mathbf{J}=\left[\begin{array}{ccc}
\mathbf{J}_{u u} & \mathbf{0} & \mathbf{J}_{u \eta} \\
\mathbf{0} & \mathbf{J}_{T T} & \mathbf{J}_{T \eta} \\
\mathbf{J}_{\eta u} & \mathbf{J}_{\eta T} & \mathbf{J}_{\eta \eta}
\end{array}\right]
$$

\section{Schur complement based preconditioners}

To maintain the strong coupling between the different physical fields involved in system (1) and at the same time use efficient solvers for the different field 

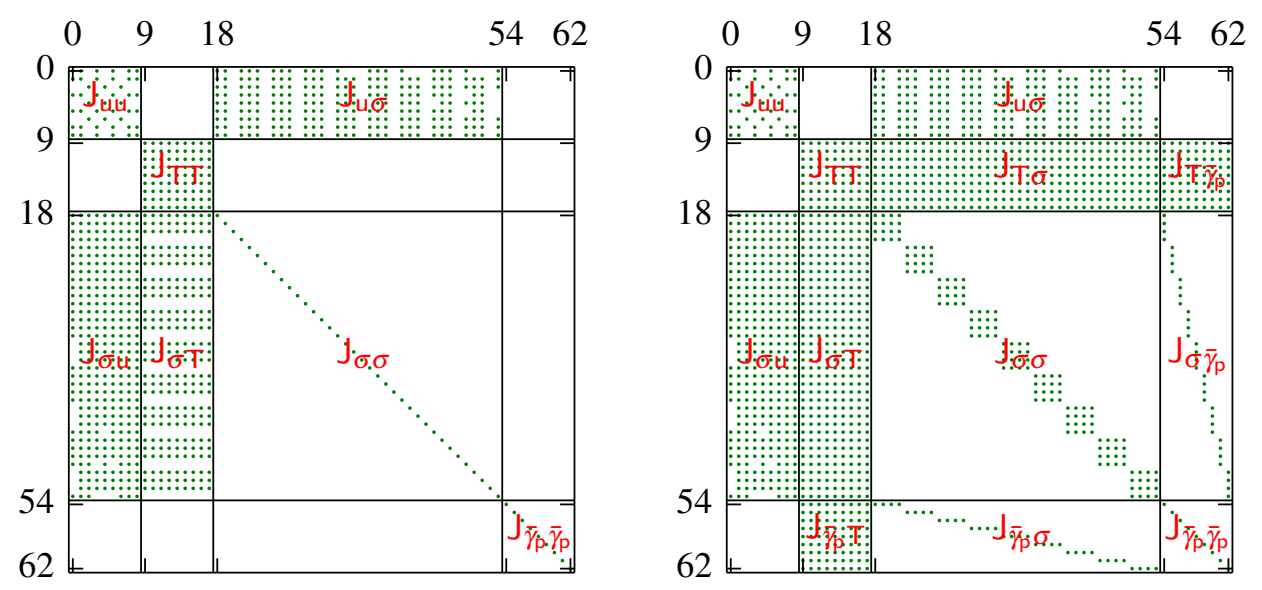

Figure 1: Sparsity pattern of a single Jacobian element matrix in the linear elastic (left) and nonlinear plastic (right) regime. The green dots indicate nonzero terms and the black lines show the limits of the blocks in the matrix.

blocks, a Schur complement method is used to precondition the GMRES solver. In the following subsections we first briefly review the Schur complement and multiplicative Schwarz methods. Next we present a special partition of the Jacobian in (6), which is used together with an outer Schur complement to derive the proposed preconditioners. The two proposed preconditioners are: (i) a Schwarz type scheme applied to the outer approximated Schur complement matrix and (ii) another inner Schur complement applied to a similar approximate of an outer Schur. For comparison, we also discuss an ilu(0) applied to the Schur complement and used as preconditioner to GMRES.

\subsection{A brief review of Schur complement method}

In the following two sections, the Schur complement method [52] is briefly introduced. Let us consider the following linear system

$$
\mathbf{J} \delta \mathbf{x}=\mathbf{R},
$$


assuming that there is a partition of the unknowns $\delta \mathbf{x}=\left[\begin{array}{l}\delta \mathbf{x}_{0} \\ \delta \mathbf{x}_{1}\end{array}\right]$ and equations $\mathbf{R}=\left[\begin{array}{l}\mathbf{R}_{0} \\ \mathbf{R}_{1}\end{array}\right]$, it is convenient to rewrite (12) in the following form

$$
\left[\begin{array}{ll}
\mathbf{J}_{00} & \mathbf{J}_{01} \\
\mathbf{J}_{10} & \mathbf{J}_{11}
\end{array}\right]\left[\begin{array}{l}
\delta \mathbf{x}_{0} \\
\delta \mathbf{x}_{1}
\end{array}\right]=\left[\begin{array}{l}
\mathbf{R}_{0} \\
\mathbf{R}_{1}
\end{array}\right]
$$

Furthermore, let us assume that the linear system is solved by first preconditioning the system with a left preconditioner. Hence, multiplying both sides by a preconditioner (matrix or linear operator) $\mathbf{P}^{-1}$, yields

$$
\mathbf{J} \delta \mathbf{x}=\mathbf{R} \quad \Rightarrow \quad \mathbf{P}^{-1} \mathbf{J} \delta \mathbf{x}=\mathbf{P}^{-1} \mathbf{R} .
$$

Two main approaches to obtain a reliable preconditioner are as follows

i. Split Preconditioners: some blocks of $\mathbf{J}$ are simplified or assumed to be zero in the computation of $\mathbf{P}$ [53]

ii. Approximate Commuter Preconditioners: some blocks of $\mathbf{J}$ are inverted using inexpensive and fast inexact algorithms [54].

The two approaches are often combined in an effort to achieve the best balance between fast, reliable and accurate preconditioners. Here we use both approaches at different stages of the proposed method. Moreover, it has been illustrated in the literature [55] that a robust strategy is to employ a GMRES solver as the iterative solver and apply these preconditioners to the Krylov space of GMRES to accelerate its convergence.

Assuming that the $\mathbf{J}_{00}$ block matrix in the partitioned system (13) is invertible, it is possible to write $\mathbf{J}$ as the product of three matrices, two of which are triangular with identity blocks on their diagonal and a block diagonal matrix, as follows

$$
\left[\begin{array}{ll}
\mathbf{J}_{00} & \mathbf{J}_{01} \\
\mathbf{J}_{10} & \mathbf{J}_{11}
\end{array}\right]=\left[\begin{array}{cc}
\mathbf{1} & \mathbf{0} \\
\mathbf{J}_{10} \mathbf{J}_{00}^{-1} & \mathbf{1}
\end{array}\right]\left[\begin{array}{cc}
\mathbf{J}_{00} & \mathbf{0} \\
\mathbf{0} & \mathbf{S}
\end{array}\right]\left[\begin{array}{cc}
\mathbf{1} & \mathbf{J}_{00}^{-1} \mathbf{J}_{01} \\
\mathbf{0} & \mathbf{1}
\end{array}\right],
$$

where

$$
\mathbf{S}=\mathbf{J}_{11}-\mathbf{J}_{10} \mathbf{J}_{00}^{-1} \mathbf{J}_{01}
$$

is the lower Schur complement of (15). The alternate expression of $\mathbf{J}$ given in equation (15) is particularly useful to compute its inverse based on the inverses of 
$\mathbf{J}_{00}$ and $\mathbf{S}$ :

$$
\begin{aligned}
\mathbf{J}^{-1} & =\left[\begin{array}{cc}
\mathbf{1} & -\mathbf{J}_{00}^{-1} \mathbf{J}_{01} \\
\mathbf{0} & \mathbf{1}
\end{array}\right]\left[\begin{array}{cc}
\mathbf{J}_{00}^{-1} & \mathbf{0} \\
\mathbf{0} & \mathbf{S}^{-1}
\end{array}\right]\left[\begin{array}{cc}
\mathbf{1} & \mathbf{0} \\
-\mathbf{J}_{10} \mathbf{J}_{00}^{-1} & \mathbf{1}
\end{array}\right] . \\
& =\left[\begin{array}{cc}
\mathbf{J}_{00}^{-1}+\mathbf{J}_{00}^{-1} \mathbf{J}_{01} \mathbf{S}^{-1} \mathbf{J}_{10} \mathbf{J}_{00}^{-1} & -\mathbf{J}_{00}^{-1} \mathbf{J}_{01} \mathbf{S}^{-1} \\
-\mathbf{S}^{-1} \mathbf{J}_{10} \mathbf{J}_{00}^{-1} & \mathbf{S}^{-1}
\end{array}\right] .
\end{aligned}
$$

This expression of the inverse of $\mathbf{J}$ shows that if $\mathbf{J}_{00}$ can be easily inverted then the computational effort can be concentrated on computing the inverse of $\mathbf{S}$. Since the cost of forming and inverting $\mathbf{S}$ is large, in practice we only compute an approximate inverse of $\mathbf{S}$ that we denote by $\mathbf{Q} \approx \mathbf{S}^{-1}$ and use it to form a preconditioner for $\mathbf{J}$. Two ways to obtain reliable $\mathbf{Q}$ operators (Schur-Schwarz and Schur-Schur methods) are presented in the following sections.

\subsection{Conservation laws-Constitutive laws split}

To invert the Jacobian matrix $\mathbf{J}$ efficiently it is crucial that the block structure of $\mathbf{J}_{\eta \eta}$ is preserved. To this end we introduce the following split: the constitutive laws, grouped in the field denoted by $\eta$, are assigned to the partition indicated by subscript 0 and the conservations laws in field $u$ and $T$ are assigned to the partition denoted by subscript 1 in Eq. (13). This split is presented in a matrix form as follow

$$
\mathbf{J}=\left[\begin{array}{c:c}
\mathbf{J}_{00} & \mathbf{J}_{01} \\
\hdashline \mathbf{J}_{10} & \mathbf{J}_{11}
\end{array}\right]=\left[\begin{array}{c:cc}
\mathbf{J}_{\eta \eta} & \mathbf{J}_{\eta u} & \mathbf{J}_{\eta T} \\
\hdashline \mathbf{J}_{u \eta} & \mathbf{J}_{u u} & \mathbf{0} \\
\mathbf{J}_{T \eta} & \mathbf{0} & \mathbf{J}_{T T}
\end{array}\right] .
$$

The sparsity pattern of the corresponding matrix is shown in Figure 2 for a single quadratic INSQ element. Note that reordering of degrees of freedom has also been applied to matrix $\mathbf{J}$ to obtain Figure 2. It is clear from the figure that the sparse structure of $\mathbf{J}_{\eta \eta}$ is preserved by this split and can then be exploited within the Schur decomposition.

With this partition of the system, the Schur complement, given in Eq.(16), that 

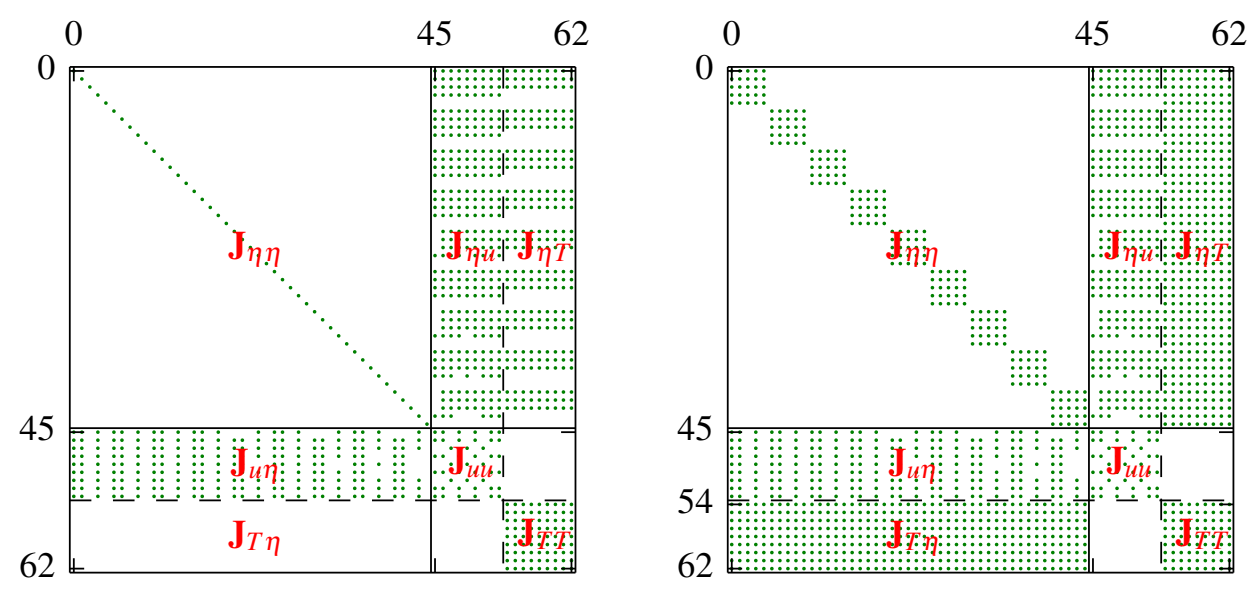

Figure 2: Sparsity pattern of the Jacobian matrix in the linear-elastic (left) and nonlinear plastic (right) regime for a single quadratic order INSQ element with 9 gauss points. The green dots indicate nonzero terms in the matrix. The solid black lines show the split of the displacementtemperature and stresses-EQPS nested blocks in the matrix, the dashed lines show the subpartition into displacement and temperature blocks.

needs to be inverted to compute $\mathbf{J}^{-1}$ is

$$
\begin{aligned}
\mathbf{S} & =\mathbf{J}_{11}-\mathbf{J}_{10} \mathbf{J}_{00}^{-1} \mathbf{J}_{01} \\
& =\left[\begin{array}{cc}
\mathbf{J}_{u u} & \mathbf{0} \\
\mathbf{0} & \mathbf{J}_{T T}
\end{array}\right]-\left[\begin{array}{l}
\mathbf{J}_{u \eta} \\
\mathbf{J}_{T \eta}
\end{array}\right] \mathbf{J}_{\eta \eta}^{-1}\left[\begin{array}{ll}
\mathbf{J}_{\eta u} & \mathbf{J}_{\eta T}
\end{array}\right] \\
& =\left[\begin{array}{cc}
\mathbf{J}_{u u}-\mathbf{J}_{u \eta} \mathbf{J}_{\eta \eta}^{-1} \mathbf{J}_{\eta u} & -\mathbf{J}_{u \eta} \mathbf{J}_{\eta \eta}^{-1} \mathbf{J}_{\eta T} \\
-\mathbf{J}_{T \eta} \mathbf{J}_{\eta \eta}^{-1} \mathbf{J}_{\eta u} & \mathbf{J}_{T T}-\mathbf{J}_{T \eta} \mathbf{J}_{\eta \eta}^{-1} \mathbf{J}_{\eta T}
\end{array}\right] \\
& =\left[\begin{array}{cc}
\mathbf{S}_{u u} & \mathbf{S}_{u T} \\
\mathbf{S}_{T u} & \mathbf{S}_{T T}
\end{array}\right] .
\end{aligned}
$$

Notice that the last term is used to simplify the notation of the schur complement. Based on equations (17) and (18) we obtain the following exact expression for the 
inverse of $\mathbf{J}$

$$
\begin{aligned}
& \mathbf{J}^{-1}=\left[\begin{array}{ccc}
\mathbf{1} & -\mathbf{J}_{\eta \eta}^{-1}\left[\begin{array}{cc}
\mathbf{J}_{\eta u} & \mathbf{J}_{\eta T} \\
\mathbf{0} & \mathbf{1}
\end{array}\right]\left[\begin{array}{cc}
\mathbf{J}_{\eta \eta}^{-1} & \mathbf{0} \\
\mathbf{0} & \mathbf{S}^{-1}
\end{array}\right]\left[\begin{array}{ccc}
\mathbf{1} & \mathbf{0} \\
\mathbf{J}_{T \eta}
\end{array}\right] \mathbf{J}_{\eta \eta}^{-1} & \mathbf{1}
\end{array}\right] \\
& =\left[\begin{array}{cc}
\mathbf{J}_{\eta \eta}^{-1}+\mathbf{J}_{\eta \eta}^{-1}\left[\begin{array}{cc}
\mathbf{J}_{\eta u} & \mathbf{J}_{\eta T}
\end{array}\right] \mathbf{S}^{-1}\left[\begin{array}{l}
\mathbf{J}_{u \eta} \\
\mathbf{J}_{T \eta}
\end{array}\right] \mathbf{J}_{\eta \eta}^{-1} & -\mathbf{J}_{\eta \eta}^{-1}\left[\begin{array}{ll}
\mathbf{J}_{\eta u} & \mathbf{J}_{\eta T}
\end{array}\right] \mathbf{S}^{-1} \\
-\mathbf{S}^{-1}\left[\begin{array}{c}
\mathbf{J}_{u \eta} \\
\mathbf{J}_{T \eta}
\end{array}\right] \mathbf{J}_{\eta \eta}^{-1} & \mathbf{S}^{-1}
\end{array}\right] .
\end{aligned}
$$

At this point the only work left is to propose an efficient and reliable strategy to compute $\mathbf{J}_{\eta \eta}^{-1}$ and $\mathbf{S}^{-1}$. Also, note that if a preconditioner is applied to a Krylovtype solver (e.g. GMRES), in order to accelerate its convergence one has to solve approximately an outer Schur complement system as follows:

$$
\mathbf{S y}=\mathbf{z}
$$

where $\mathbf{z}$ is an incoming component of a Krylov vector and $\mathbf{y}$ is the preconditioned outgoing vector. Furthermore, solving approximately Eq. (21) can also be accelerated by a preconditioning scheme on its own, as follows

$$
\overline{\mathbf{P}}^{-1} \mathbf{S y}=\overline{\mathbf{P}}^{-1} \mathbf{z}
$$

where $\overline{\mathbf{P}}^{-1}$ is a preconditioner used for the Schur complement sub problem. However, two difficulties arise when attempting to solve system (21) or (22): (i) $\mathbf{S}$ itself is computationally expensive to be formed explicitly and (ii) since $\mathbf{S}$ cannot be formed exactly, $\overline{\mathbf{P}}$ cannot be directly obtained from $\mathbf{S}$ which limits the choice of available preconditioners for the Schur complement problem.

To this end, in order to resolve difficulty (ii) and form a preconditioner, we approximate $\mathbf{S}$ by $\mathbf{S}^{*}$ that has the following expression

$$
\begin{aligned}
\mathbf{S} \approx \mathbf{S}^{*} & =\mathbf{J}_{11}-\mathbf{J}_{10}\left[\operatorname{diag}\left(\mathbf{J}_{00}\right)\right]^{-1} \mathbf{J}_{01} \\
& =\left[\begin{array}{ll}
\mathbf{J}_{u u} & \mathbf{J}_{u T} \\
\mathbf{J}_{T u} & \mathbf{J}_{T T}
\end{array}\right]-\left[\begin{array}{l}
\mathbf{J}_{u \eta} \\
\mathbf{J}_{T \eta}
\end{array}\right]\left[\operatorname{diag}\left(\mathbf{J}_{\eta \eta}\right)\right]^{-1}\left[\begin{array}{ll}
\mathbf{J}_{\eta u} & \mathbf{J}_{\eta T}
\end{array}\right] \\
& =\left[\begin{array}{ll}
\mathbf{S}_{u u}^{*} & \mathbf{S}_{u T}^{*} \\
\mathbf{S}_{T u}^{*} & \mathbf{S}_{T T}^{*}
\end{array}\right]
\end{aligned}
$$

where the diagonal part of $\mathbf{J}_{\eta \eta}$ is employed and $\mathbf{S}^{*}$ can be easily formed. Now the preconditioner $\overline{\mathbf{P}}^{-1}$ is formed based on $\mathbf{S}^{*}$ and the preconditioned Schur complement system is written as

$$
\left[\overline{\mathbf{P}}\left(\mathbf{S}^{*}\right)\right]^{-1} \mathbf{S y}=\left[\overline{\mathbf{P}}\left(\mathbf{S}^{*}\right)\right]^{-1} \mathbf{z} .
$$


We emphasize that Eq. (24) need only be solved approximately, for example, a few inner iterations using Richardson's method may suffice. Hence, an optimal performance can be achieved by considering the optimal number of inner and outer iterations which would lead to a minimal solution wall time. In the subsequent part of this paper $\mathbf{Q}$ will denote the approximate inverse of $\mathbf{S}$ which will either be obtained by a simplification of the expression of $\mathbf{S}$ or by an inexact linear solve used to compute $\mathbf{S}^{-1}$.

\subsection{The Schur-Schwarz and Schur-Schur preconditioners}

The first straightforward option to obtain $\mathbf{Q}$ is by performing a few iterations of a Krylov-type method such as GMRES with an ILU(0) preconditioner applied to Eq. (24). In this case $\overline{\mathbf{P}}_{i l u}$ is the ILU(0) preconditioner obtained from $\mathbf{S}^{*}$. While this is an obvious choice, it is shown later that it is not a good one. The systems arising in the shear band problem have strong coupling between components, in particular when plasticity initiates and the shear band starts to form, and hence such preconditioners yields poor performance.

A better choice for $\mathbf{Q}$ is a multiplicative Schwarz method [56]. See Appendix A for a detailed description of the method. In this approach, one defines first the following block diagonal matrices

$$
\mathbf{B}_{0}=\left[\begin{array}{cc}
\mathbf{S}_{u u}^{*-1} & \mathbf{0} \\
\mathbf{0} & \mathbf{1}
\end{array}\right] \quad \text { and } \quad \mathbf{B}_{1}=\left[\begin{array}{cc}
\mathbf{1} & \mathbf{0} \\
\mathbf{0} & \mathbf{S}_{T T}^{*-1}
\end{array}\right],
$$

then the preconditioner is obtained by setting

$$
\begin{aligned}
\overline{\mathbf{P}}_{s z}^{-1} & =\mathbf{B}_{0}+\mathbf{B}_{1}-\mathbf{B}_{1} \mathbf{S}^{*} \mathbf{B}_{0} \\
& =\left[\begin{array}{cc}
\mathbf{S}_{u u}^{*-1} & \mathbf{0} \\
\mathbf{0} & \mathbf{1}
\end{array}\right]+\left[\begin{array}{cc}
\mathbf{1} & \mathbf{0} \\
\mathbf{0} & \mathbf{S}_{T T}^{*-1}
\end{array}\right]-\left[\begin{array}{cc}
\mathbf{1} & \mathbf{0} \\
\mathbf{0} & \mathbf{S}_{T T}^{*-1}
\end{array}\right]\left[\begin{array}{cc}
\mathbf{S}_{u u}^{*} & \mathbf{S}_{u T}^{*} \\
\mathbf{S}_{T u}^{*} & \mathbf{S}_{T T}^{*}
\end{array}\right]\left[\begin{array}{cc}
\mathbf{S}_{u u}^{*-1} & \mathbf{0} \\
\mathbf{0} & \mathbf{1}
\end{array}\right] \\
& =\left[\begin{array}{cc}
\mathbf{S}_{u u}^{*-1} & -\mathbf{S}_{u T}^{*} \\
-\mathbf{S}_{T T}^{*-1} \mathbf{S}_{T u}^{*} \mathbf{S}_{u u}^{*-1} & \mathbf{S}_{T T}^{*-1}
\end{array}\right]
\end{aligned}
$$

In this case we apply only one iteration of a Richardson method to Eq. (24), hence the overall action of $\mathbf{P}_{s z}^{-1}$ (in this case $\mathbf{Q}_{s z}=\overline{\mathbf{P}}_{s z}^{-1}$ ) can be written as

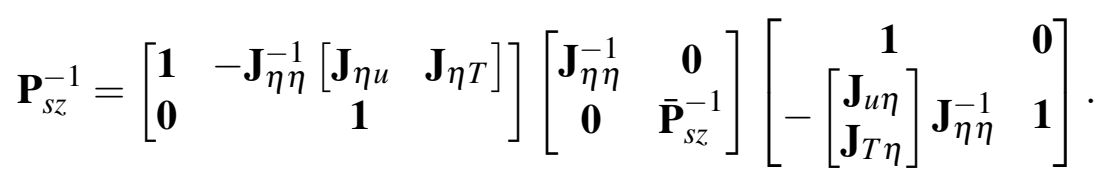


Finally the block $\mathbf{S}_{u u}^{*-1}$ and $\mathbf{S}_{T T}^{*-1}$ in $\overline{\mathbf{P}}_{s z}^{-1}$, are inverted using an LU direct solver and a fully converged GMRES solver preconditioned by ILU, respectively.

The second proposed preconditioner is the so called Schur-Schur preconditioner, denoted $\mathbf{P}_{s r}$. The key idea is to decompose the outer Schur sub problem, given in Eq. (21) with a second Schur complement method, written as

$$
\mathbf{S}^{*}=\left[\begin{array}{ll}
\mathbf{S}_{u u}^{*} & \mathbf{S}_{u T}^{*} \\
\mathbf{S}_{T u}^{*} & \mathbf{S}_{T T}^{*}
\end{array}\right]=\left[\begin{array}{cc}
\mathbf{1} & \mathbf{0} \\
\mathbf{S}_{T u}^{*} \mathbf{S}_{u u}^{*-1} & \mathbf{1}
\end{array}\right]\left[\begin{array}{cc}
\mathbf{S}_{u u}^{*} & \mathbf{0} \\
\mathbf{0} & \mathbf{S}_{\text {inner }}
\end{array}\right]\left[\begin{array}{cc}
\mathbf{1} & \mathbf{S}_{u u}^{*-1} \mathbf{S}_{u T}^{*} \\
\mathbf{0} & \mathbf{1}
\end{array}\right]
$$

where $\mathbf{S}_{\text {inner }}$ is

$$
\mathbf{S}_{\text {inner }}=\mathbf{S}_{T T}^{*}-\mathbf{S}_{T u}^{*} \mathbf{S}_{u u}^{*-1} \mathbf{S}_{u T}^{*} .
$$

Following Eq. (17) the exact inverse for the outer Schur complement reads

$$
\mathbf{S}^{*-1}=\left[\begin{array}{cc}
\mathbf{1} & -\mathbf{S}_{u u}^{*-1} \mathbf{S}_{u T}^{*} \\
\mathbf{0} & \mathbf{1}
\end{array}\right]\left[\begin{array}{cc}
\mathbf{S}_{u u}^{*-1} & \mathbf{0} \\
\mathbf{0} & \mathbf{S}_{\text {inner }}^{-1}
\end{array}\right]\left[\begin{array}{cc}
\mathbf{1} & \mathbf{0} \\
-\mathbf{S}_{T u}^{*} \mathbf{S}_{u u}^{*-1} & \mathbf{1}
\end{array}\right]
$$

Hence, to form a Schur-Schur type preconditioner to $\mathbf{S}^{-1}$, we first need to obtain an approximation for the inner Schur block. To this end, the same approximation as the one in Eq. (23) is proposed

$$
\mathbf{S}_{\text {inner }} \approx \mathbf{S}_{\text {inner }}^{*}=\mathbf{S}_{T T}^{*}-\mathbf{S}_{T u}^{*}\left[\operatorname{diag}\left(\mathbf{S}_{u u}^{*}\right)\right]^{-1} \mathbf{S}_{u T}^{*} .
$$

The inner block $\mathbf{S}_{\text {inner }}^{*}$ is approximated by one iteration of an ILU(0) solver. This approach works well due to the fact the $\mathbf{S}_{\text {inner }}^{*}$ is the result of two nested Schur complements, which yields a dense and concentrated sparsity pattern, where an ILU(0) is a good approximation of a direct solve. In summary, this preconditioner includes two levels of Schur decomposition with a few approximations along the way. Hence we obtain the following abstract expression for the Schur-Schur pre- 
conditioner

$$
\begin{aligned}
& \mathbf{P}_{s r}^{-1}=\left[\begin{array}{ccc}
\mathbf{1} & -\mathbf{J}_{\eta \eta}^{-1}\left[\begin{array}{cc}
\mathbf{J}_{\eta u} & \mathbf{J}_{\eta T} \\
\mathbf{0} & \mathbf{1}
\end{array}\right]\left[\begin{array}{cc}
\mathbf{J}_{\eta \eta}^{-1} & \mathbf{0} \\
\mathbf{0} & \mathbf{Q}_{s r}
\end{array}\right]\left[\begin{array}{cc}
\mathbf{1} & \mathbf{0} \\
\mathbf{J}_{T \eta}
\end{array}\right] \mathbf{J}_{\eta \eta}^{-1} & \mathbf{1}
\end{array}\right] \\
& =\left[\begin{array}{cc}
\mathbf{1} & -\mathbf{J}_{\eta \eta}^{-1}\left[\begin{array}{cc}
\mathbf{J}_{\eta u} & \mathbf{J}_{\eta T}
\end{array}\right] \\
\mathbf{0} & \mathbf{1}
\end{array}\right]
\end{aligned}
$$

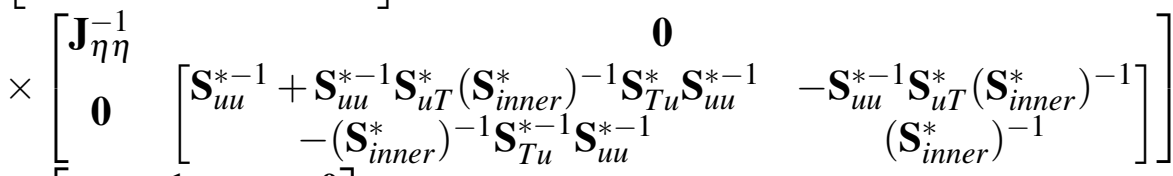

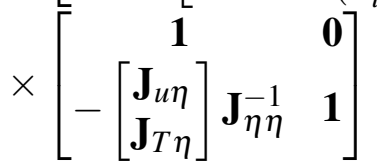

where again, $\mathbf{Q}$ denotes the two level preconditioner that is acting on the outer global system. The approximate inverse of $\mathbf{S}_{\text {inner }}^{*}$ is denoted by $\left(\mathbf{S}_{\text {inner }}^{*}\right)^{-1}$ in equation (32) even though it is not an exact inverse. As with the Schur-Schwarz preconditioner, the $\mathbf{S}_{u u}^{*-1}$ block is inverted using an LU solver.

The choice of an LU solver for the $\mathbf{S}_{u u}^{*}$ matrix is justified as its condition number is at least an order of magnitude higher than the other blocks and degrades as a function of the deformation process and the appearance of the shear band.

To observe the effectiveness of the Schur-Schwarz and Schur-Schur preconditioners, the iteration matrix defined as follows

$$
\mathbf{M}_{\text {iter }}=\mathbf{1}-\mathbf{P}^{-1} \mathbf{J}
$$

is computed for each of the three preconditioners and its eigenvalues are plotted and illustrated in Figure 3. Note that we have considered one INSQ element for this illustration. As can be observed, most eigenvalues fall within the unit circle which ensures a rapid reduction of most components of the error. The eigenvalues that do not fall in the unit circle represent modes that are amplified by the application of $\mathbf{P}^{-1}$. As the simulation progresses and plasticity develops, the off diagonal terms of $\mathbf{J}_{00}$ become more prominent, and several modes are found to lie outside the unit circle starting at around $0.2 \mu s$ of the simulation. The magnitude of these divergent modes increases later in the simulation as the plastic straining becomes more intense.

Complete descriptions of the Schur-Schwarz and Schur-Schur preconditioners with all block solvers and approximations specified are provided in the flow chart presented in Figure 4. 

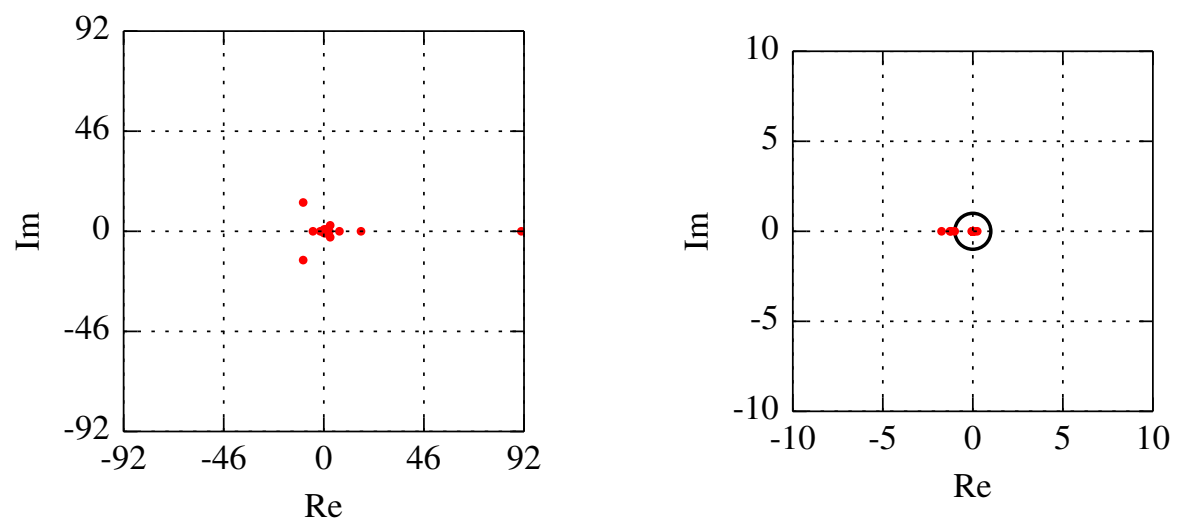

Figure 3: Eigenvalues of $\mathbf{P}_{s z}$ (left) and $\mathbf{P}_{s r}$ (right). Note that the scale of the axis is changing due to the large eigen values of $\mathbf{P}_{s z}$. $\mathbf{P}_{s z}$ has 14 eigen values larger than 1 and $\mathbf{P}_{s r}$ has 4 eigen values larger than 1 . Note that the effectiveness of the preconditioner varies as the system evolves with time and the shear band is formed.

\section{Numerical results}

In this section we demonstrate the capabilities and performance of the proposed preconditioners on a $45^{\circ}$ shear band benchmark example. In the first section the parameters of the problem are presented, in the second and third sections two sets of numerical studies are presented:

- Serial studies: using IsoGeometric Analysis (NURBS type basis functions) [31] to observe the behavior of the preconditioners as the shear band initiates and propagates and the effect of $k$ - and $h$-refinement on the convergence of the preconditioners,

- Parallel studies: using standard irreducible bilinear elements [29, 30, 57] to observe the parallel performance of the preconditioners.

The first series of numerical examples, involve smaller meshes and high order discretizations, are run on a DELL optiplex 755 with 4 cores at $2.83 \mathrm{GHz}$ and $4 \mathrm{~GB}$ of RAM. The second series of numerical examples are run using the MIRA cluster $[58,59]$, which is part of the Argonne Leadership Computing Facility (ALCF), at Argonne National Labs. The problem is partitioned into processors using the library METIS/PARMETIS [60,61] and the PDE model is implemented using 


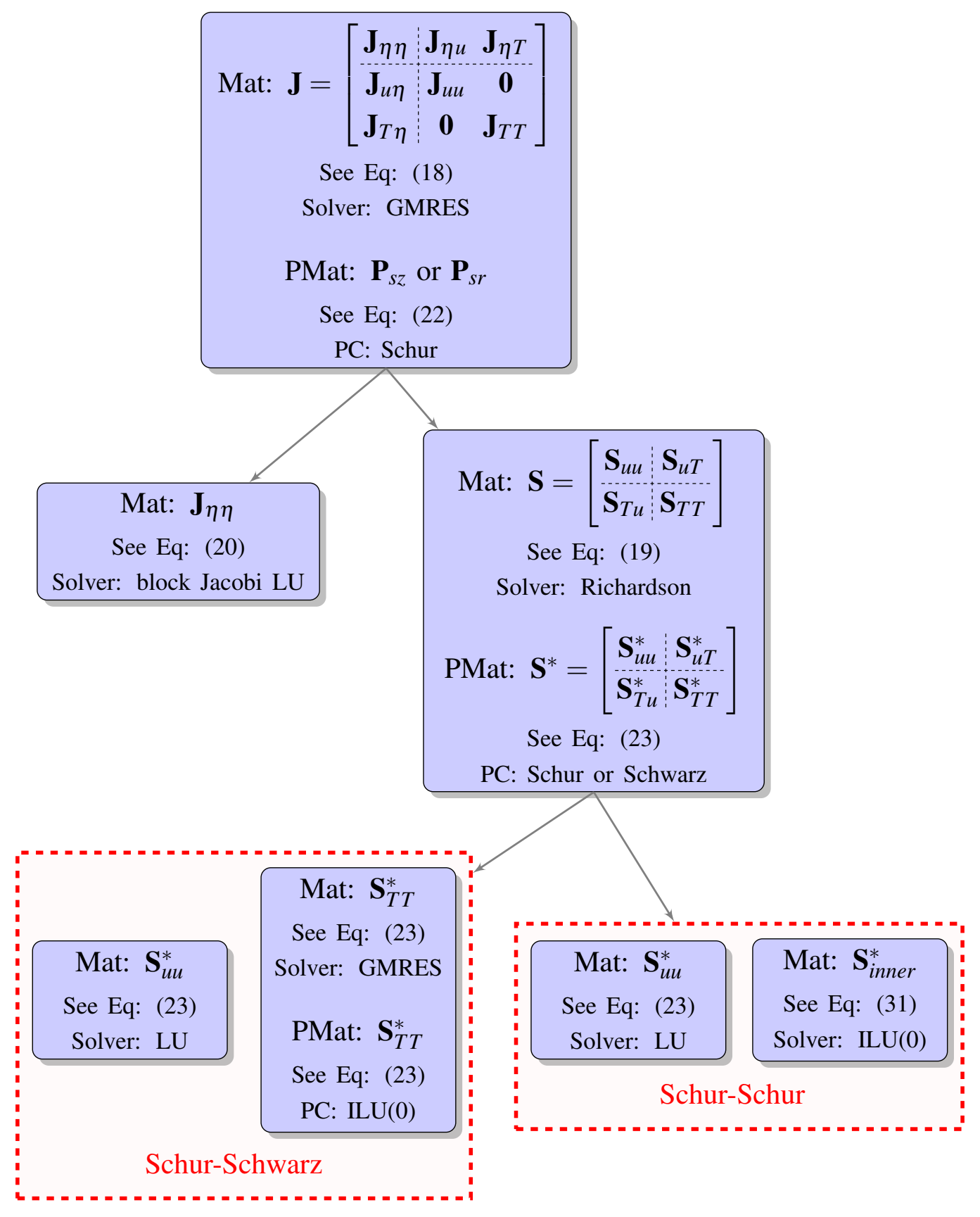

Figure 4: Flow chart of the Schur-Schwarz and Schur-Schur preconditioners, Mat indicates the matrix being solved, Solver indicates if direct or iterative solver is used. If a preconditioner is applied, PMat denotes the matrix used to construct the preconditioner and PC indicates the type of preconditioner. 
the Finite Element Analysis Program (FEAP) [62]. The linear solves are done by calling the FieldSplit option in PETSc $[63,64,65]$ and using the external packages MUMPS [66] and Hypre Euclid [67]. The posprocessing and graphics are generated using ParaView [68] and Matplotlib [69].

\subsection{The $45^{\circ}$ shear band problem}

Consider a square plate under pure tension applied at the top and bottom edges. The plate has a circular imperfection at its center that triggers the localization of the deformation into two shear bands forming an X-shape passing to the four corners of the plate through its center. The imperfection employed reduces the value of the yield stress and yield strain using a 2D beta function see Figure 5 given as

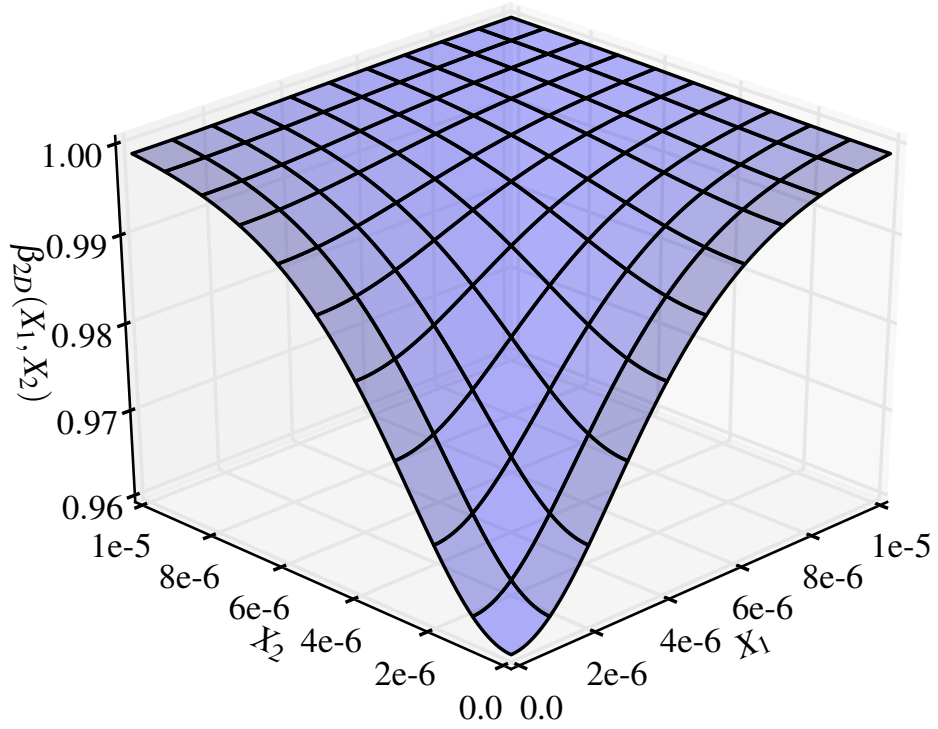

Figure 5: Two dimensional $\beta$ function used to model a material imperfection centered at $\left(X_{1}, X_{2}\right)=$ $(0,0)$.

$$
\sigma_{\text {yield }}=\sigma_{0} \beta_{2 D}(x, y), \quad \gamma_{\text {yield }}=\gamma_{0} \beta_{2 D}(x, y)
$$


with

$$
\beta_{2 D}(x, y)=1-0.04\left[\operatorname{sech}\left(\frac{\sqrt{x^{2}+y^{2}}}{10^{-5}}\right)\right]^{2} .
$$

This corresponds to a smooth imperfection centered at $x=0$ and $y=0$ with a maximal reduction of reference yield parameters by $4 \%$. Due to the symmetries of this problem only a quarter of the plate needs to be modeled, herein the top right quarter of the plate. The width of the quarter plate is $10 \mu \mathrm{m}$ and a uniform velocity $v_{B C}$ is applied at its top edge. $v_{B C}$ increases linearly from $v_{B C}=0 \mathrm{~m} / \mathrm{s}$ to $v_{B C}=5 \mathrm{~m} / \mathrm{s}$ from time $t=0$ to time $t=1 \mu \mathrm{s}, v_{B C}$ remains constant afterward. A null thermal flux condition is applied to all the edges of the plate. The initial values of displacement, stresses and plastic strains are taken as zero and the initial temperature is set to $T=293.0 \mathrm{~K}$ (room temperature). The values of the material parameters used to run the simulations are presented in Table B.9 in Appendix B. The profile of $v_{B C}$ and a schematic representation of the plate are shown in Figure 6.

The stress-strain and stress-time curves, averaged over the plate, are recorded and shown in Figure 7. The vertical lines in the figure indicate the three stages of the deformation (linear elastic, onset of plasticity and stress collapse) for which the Jacobian matrix will change. The corresponding plastic strain field and the specific sparsity pattern of the Jacobian are shown in Figure 8. Note that the sparsity pattern changes with the plasticity in the system and the values in the matrix increase significantly, which indicate a high degree of nonlinearity and coupling between the physics.

In general the number of equations in the problem is given by

$$
n_{e q}=3 \times n_{n}+5 \times n_{e l} \times n_{g p}-n_{b c}
$$

where $n_{n}$ is the number of nodes in the problem, $n_{e l}$ is the number of elements in the problem, $n_{g p}=(p+1)^{2}$ is the number of gauss points per elements, $p$ is the polynomial order of the shape functions and $n_{b c}$ is the number of Dirichlet boundary conditions applied to the problem.

In the simulations presented in the results section we only consider uniform structured meshes with equal number of nodes in both $x$ and $y$ directions and Dirichlet boundary conditions are applied along three edges of the plate. This simplifies expression (36) to

$$
n_{e q}=\left(5(p+1)^{2}+3\right) n^{2}+3 n
$$

where $n$ is the number of elements per spatial direction.

The PDE system is integrated in time, where at every time step, a monolithic 


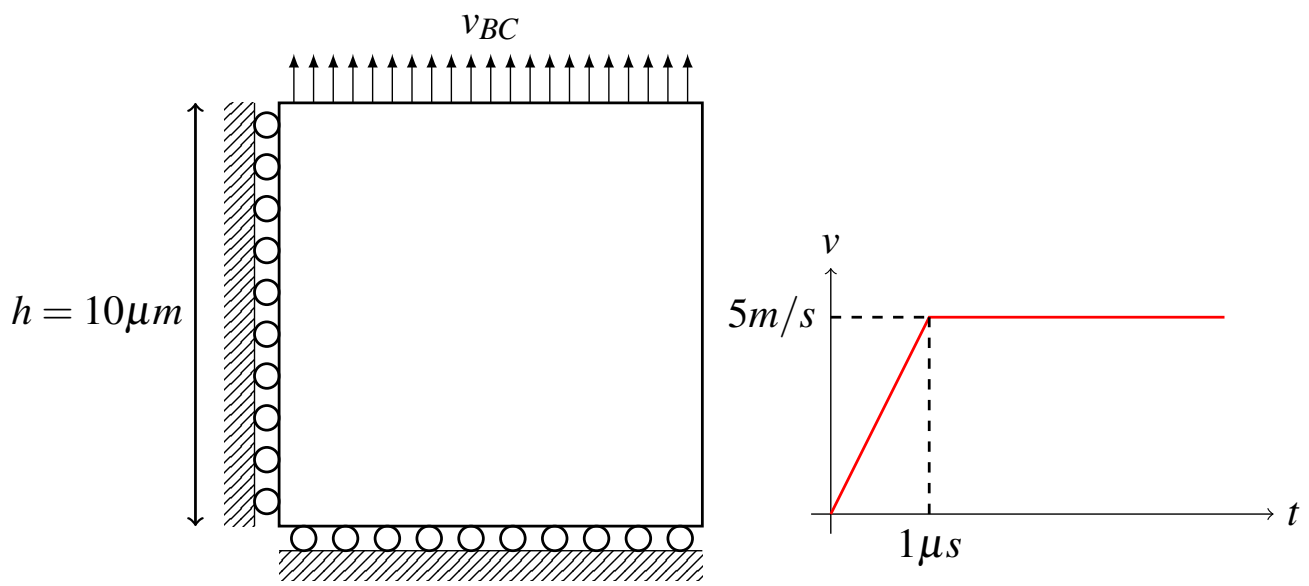

Figure 6: The modeled quarter plate with it boundary conditions and the evolution of the velocity $v_{B C}$.

solution is obtained using a Newton method. Newton iterations are terminated under the following conditional norms:

- Relative residual: $\frac{\left\|\mathbf{R}_{\beta, n+1}^{\alpha}\right\|}{\left\|\mathbf{R}_{\beta, n}\right\|} \leq 10^{-4}$

- Absolute residual: $\left\|\mathbf{R}_{\beta, n+1}^{\alpha}\right\| \leq 10^{-12}$

- Relative solution increment: $\frac{\left\|\delta x_{\beta, n+1}^{\alpha}\right\|}{\left\|\delta x_{\beta, n}\right\|} \leq 10^{-16}$

where $\beta$ is the set of all multiphysics degrees of freedom, corresponding to $\forall \beta \in$ $\left\{u, T, \sigma, \bar{\gamma}_{p}\right\} . n+1$ denotes the current time step and $\alpha$ denotes the current Newton iteration. These criterions ensure that a correct solution for each field is obtained at every time step.

The convergence criteria for the GMRES linear solver applied to the global system in Eq. (12) is

$$
\frac{\left\|\mathbf{R}_{j}\right\|}{\left\|\mathbf{R}_{0}\right\|} \leq 10^{-8}
$$

where $j$ denotes the current linear solver iteration.

In Table 1 we present the evolution of the condition number of several matrices involved in the computations during the solution phase for two meshes: $10 \times 10$ elements (Jacobian size of 4963) and $20 \times 20$ elements (Jacobian size of 9323). It 

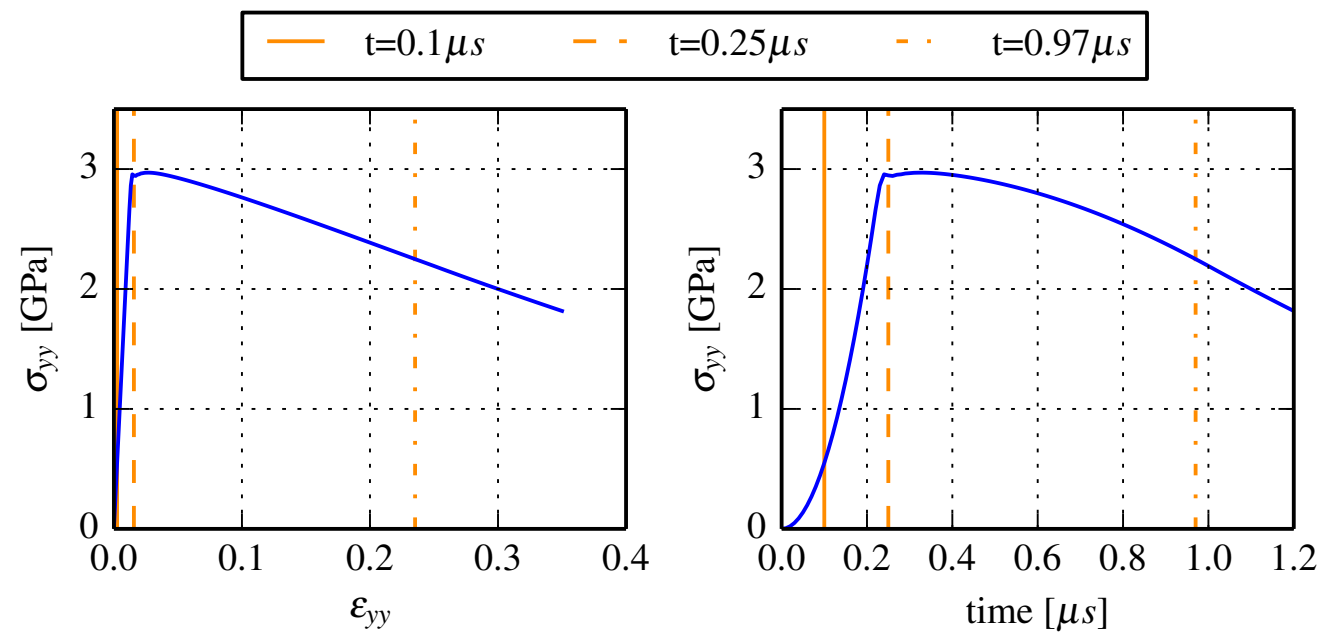

Figure 7: Stress-strain and stress-time curves for the $45^{\circ}$ shear band problem.

can be observed that the Jacobian matrix has a condition number that is both sensitive to the onset of localization (mild plasticity) and to the stress collapse regime (high plasticity and nonlinearity). The $\mathbf{S}_{u u}^{*}$ is not too sensitive to the onset of localization since it mainly represents the displacement field of an approximate Schur complement. However it is strongly sensitive in the stress collapse regime as near rigid body modes appear due to the softening that takes place. The thermal blocks $\mathbf{S}_{T T}^{*}$ and $\mathbf{S}_{\text {inner }}^{*}$ are the most stable since those are not affected by near rigid body modes. It also interesting to note that the Schur complement matrix $\mathbf{S}$ results in the highest condition number, orders of magnitude larger than all other matrices.

\subsection{Serial performance of the preconditioners applied to Isogeometric discretiza- tions}

In this section the problem presented in Figure 6 is descretized using the INSQ element [31] that uses NURBS based shape functions for displacements and temperature fields, while the stresses and plastic strain are gauss point history variables. This element has been shown to have good convergence properties upon hrefinement and k-refinement but the latest leads to increased computational times with a classic direct LU solver. With NURBS shape functions of degree $p \geq 1$ the preconditioners that are proposed are facing a more complex task since the coupling in $\mathbf{J}_{u u}$ and in $\mathbf{J}_{T T}$ are occurring between larger numbers of degrees of 

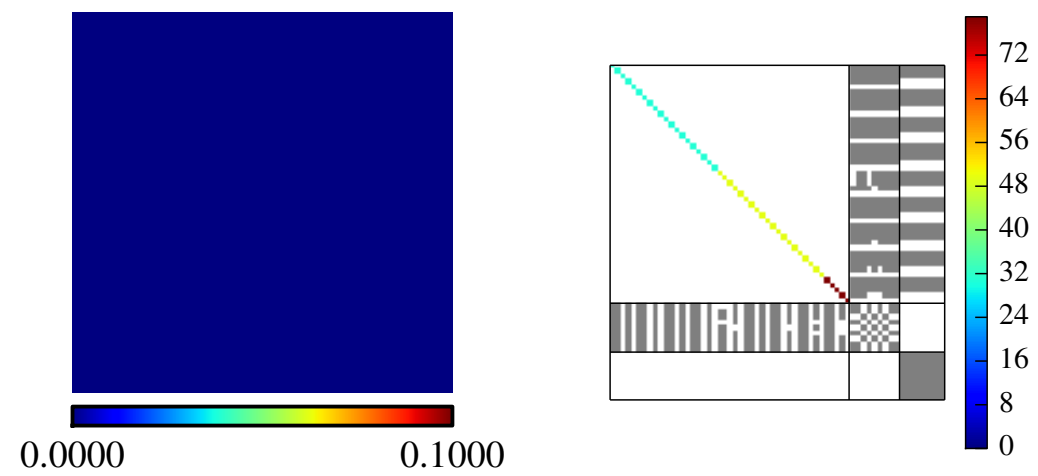

(a) EQPS field (left) and element matrix (right) at $\varepsilon_{y y}=2.5 e^{-3}$ and $t=0.10 \mu \mathrm{s}$, corresponding to the linear elastic regime.
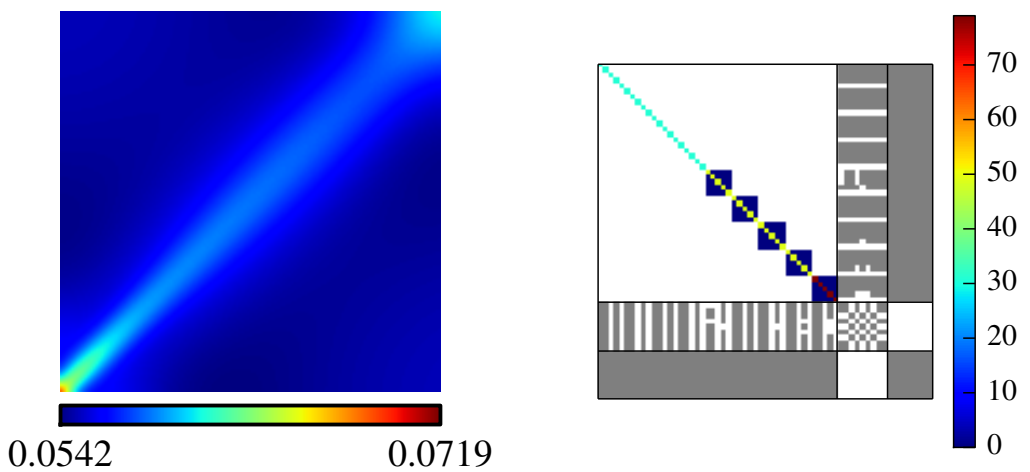

(b) EQPS field (left) and element matrix (right) at $\varepsilon_{y y}=1.6 e^{-2}$ and $t=0.25 \mu \mathrm{s}$, corresponding to the onset of localization (which indicates mild nonlinearity).
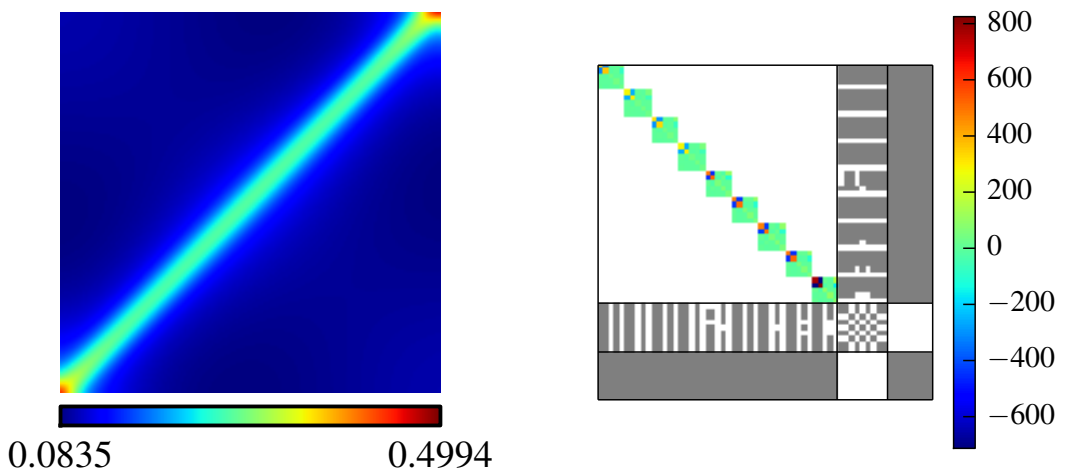

(c) EQPS field (left) and element matrix (right) at $\varepsilon_{y y}=2.4 e^{-1}$ and $t=0.97 \mu \mathrm{s}$, corresponding to the the stress collapse regime (which indicates high nonlinearity).

Figure 8: EQPS and Jacobian element sparsity pattern for quadratic (P2) INSQ element, corresponding to the three deformation regimes marked in the stress-strain and stress-time curves of Figure 7. We purposely highlight only the $\mathbf{J}_{\eta \eta}$ terms where the color bar indicate the values in this block. Notice that the sparsity pattern of the Jacobian is evolving with the plasticity present in the system. 


\begin{tabular}{|c|c|c|c|c|c|c|c|}
\hline & \multicolumn{2}{|c|}{ Linear elastic } & \multicolumn{2}{|c|}{ Onset localization } & \multicolumn{2}{|c|}{ Stress collapse } & \multirow[t]{2}{*}{ Solvers choice } \\
\hline & $10 \times 10$ & $20 \times 20$ & $10 \times 10$ & $20 \times 20$ & $10 \times 10$ & $20 \times 20$ & \\
\hline $\mathbf{J}$ & $9.18 \mathrm{e}+3$ & $1.82 \mathrm{e}+4$ & $1.06 \mathrm{e}+4$ & $2.13 \mathrm{e}+4$ & $1.31 \mathrm{e}+4$ & $2.85 \mathrm{e}+4$ & ${\overline{G M R E S}+\mathbf{P}_{s}}_{s}$ \\
\hline $\mathbf{S}$ & $2.05 \mathrm{e}+6$ & $6.28 \mathrm{e}+6$ & $1.64 \mathrm{e}+6$ & $4.92 \mathrm{e}+6$ & $1.69 \mathrm{e}+7$ & $3.96 e+7$ & Richardson $+\overline{\mathbf{P}}_{s r}$ or $\overline{\mathbf{P}}_{S z}$ \\
\hline $\mathbf{S}_{u u}^{*}$ & $1.86 \mathrm{e}+2$ & $6.93+2$ & $1.96 \mathrm{e}+2$ & $7.82+2$ & $5.04 \mathrm{e}+2$ & $5.62 \mathrm{e}+3$ & LU \\
\hline $\mathbf{S}_{T T}^{*}$ & $7.43 e+0$ & $2.13 \mathrm{e}+1$ & $7.29 \mathrm{e}+0$ & $2.02 \mathrm{e}+1$ & $6.74 \mathrm{e}+0$ & $1.98 \mathrm{e}+1$ & GMRES+ILU(0) \\
\hline $\mathbf{S}_{\text {inner }}^{*}$ & $7.43 e+0$ & $2.13 e+1$ & $7.35 \mathrm{e}+0$ & $2.03 \mathrm{e}+1$ & $6.89 \mathrm{e}+0$ & $1.98 \mathrm{e}+1$ & $\operatorname{ILU}(0)$ \\
\hline
\end{tabular}

Table 1: Condition number of the Jacobian, Schur complement and approximate Schur blocks $\mathbf{S}_{u u}^{*}, \mathbf{S}_{T T}^{*}$ and $\mathbf{S}_{\text {inner }}^{*}$ matrices at $t=0.10 \mu s$ (linear elastic), $t=0.25 \mu s$ (onset of localization) and $t=0.97 \mu \mathrm{s}$ (stress collapse). A detailed discussion on solvers and preconditioners has been presented in Section 3

freedom than with regular bilinear shape functions.

One noticeable aspect with the implementation of the proposed preconditioners is the memory allocations requirements. Since LU direct solvers are used for comparison as well as to solve inner subblocks in each of the preconditioners, as shown in Figure 4, it is important to make optimal choices for LU solvers. Accurate identification of the sparsity pattern of each element leads to more efficient memory allocations and therefore significant cpu time savings. Table 2 illustrates the gains in memory usage and computational time of sparse element memory allocation in comparison with dense element memory allocation. These results are reported for on a 30 by 30 mesh with quadratic (P2-NURBS) elements for a simulation running from $t=0$ to $t=1.2 \mu \mathrm{s}$. It can be observed that about $20 \%$ savings in memory allocation directly leads to $20 \%$ savings in cpu time for the proposed preconditioners. However, describing accurately the sparsity pattern of the element matrix, may also lead to highly degraded performance of LU solvers. This degradation can be minimized if a proper reordering of equations algorithm is employed. Optimal LU results are obtained with the Nested Dissection algorithm [70, 71], which is the default in PETSc, for dense memory allocations. However, for sparse element allocations using a Minimum Degree ordering (AMD or QMD) [72, 73] which gives better timings. The results in the Table 2 have been obtained using the optimal ordering for each element allocation type.

Figure 9a shows the stress-strain curve similar to Figure 7 and in addition the number of linear iterations of GMRES solver at the first Newton iteration of each time step. Due to the choice of a relatively small time step, $\Delta t=40 \mathrm{~ns}$, the number of Newton iterations per time step does not exceed 6. Nonetheless the maximum 


\begin{tabular}{c|c|c|c|c} 
& \multicolumn{2}{|c|}{ Dense element allocation } & \multicolumn{2}{c}{ Sparse element allocation } \\
& cpu [s] & Memory [MB] & cpu [s] & Memory [MB] \\
\hline LU solver & 323.82 & 150.83 & 448.67 & 128.11 \\
Schur-Schwarz & 427.94 & 195.94 & 413.32 & 170.18 \\
Schur-Schur & 568.24 & 194.98 & 382.93 & 170.13
\end{tabular}

Table 2: Comparison of cpu time [s] (wall time of the simulation) for dense element allocation and sparse element allocation of the Jacobian matrix on a 30 by 30 mesh with quadratic elements from $t=0$ to $t=1.2 \mu \mathrm{s}$.

number of Newton iterations occur at the onset of plasticity $\left(\varepsilon_{y y}=0.0025\right)$ and rapidly reduces back to three or two iterations per time step. On the other hand, the linear iterations required to converge at each Newton iteration increase steadily with the increase of the deformation, as indicated in Figure 9b. This is due to the increasing spectral radius of the iteration matrix as shown in Figure 3 and the evolving sparsity pattern of the Jacobian matrix, as illustrated in Figure 8.

Table 3 reports a comprehensive convergence study of the preconditioners with respect to h- and k-refinements using the NURBS shape functions. The problem described in Figure 6 is solved using four different meshes: $10 \times 10,20 \times 20$, $30 \times 30$ and $40 \times 40$ elements, with four different NURBS shape functions degree: $p=1, p=2, p=3$ and $p=4$.

As can be expected the proposed preconditioners have larger costs than LU solvers on small systems because of the setup time required for the Schur-Schwarz and Schur-Schur preconditioners. We also observe that the Schur-Schwarz preconditioner is more suited for low order discretizations outperforming the SchurSchur preconditioner on almost all P1 meshes. However, as the polynomial order is increased the Schur-Schur preconditioner is clearly more efficient than the Schur-Schwarz perconditioner. Note also that an off-the-shelf Schur-ILU preconditioner is clearly the worst preconditioner with the poorest performance.

\subsection{Parallel performance of the preconditioners}

In this section we study the convergence and scalability of the preconditioners implemented on a parallel machine (MIRA) at Argonne National Lab. The supercomputer MIRA used for these simulations is composed of 49,512 compute nodes, each node has $16 \mathrm{~GB}$ of dedicated shared RAM and 16 Power PC A2 cores at $1600 \mathrm{MHz}$. 


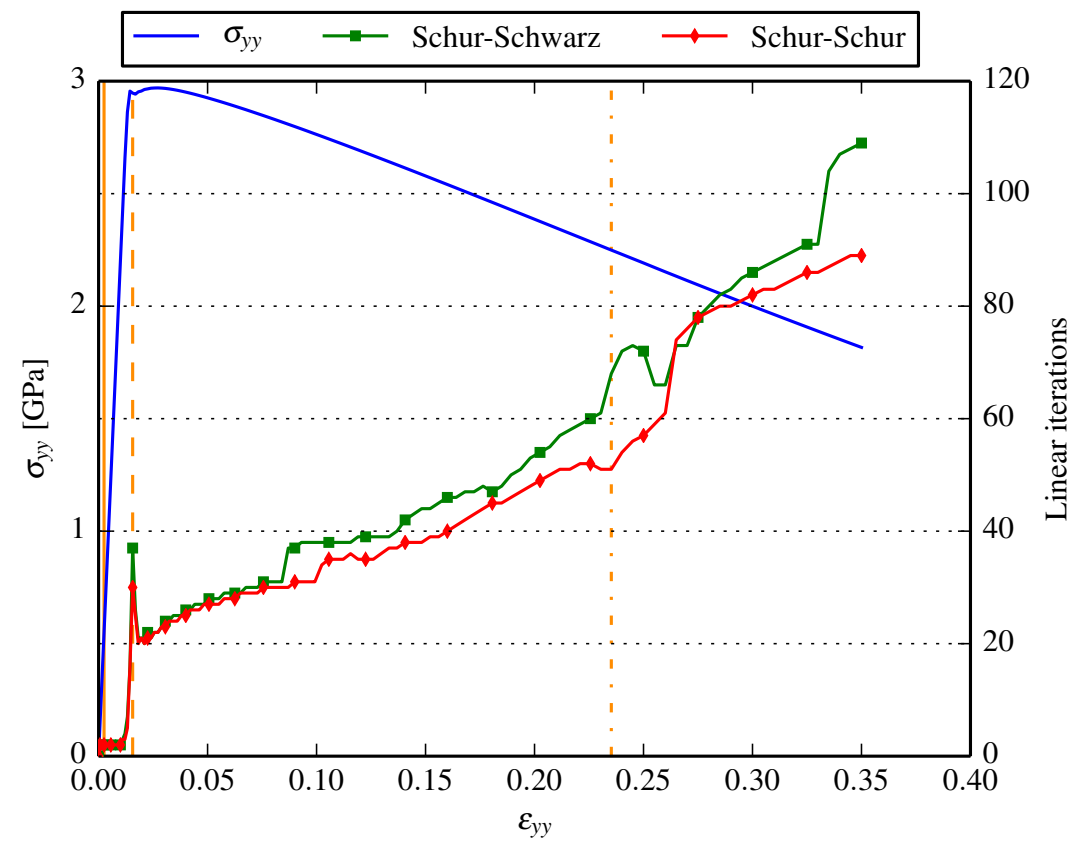

(a) Stress-strain curve and outer GMRES iterations obtained for a 20 by 20 element mesh with P3 NURBS discretization. The linear iterations are shown for the system that arise at the first Newton iteration at every time step. The three vertical lines mark time steps in the linear regime, the onset of plasticity and the softening regime.

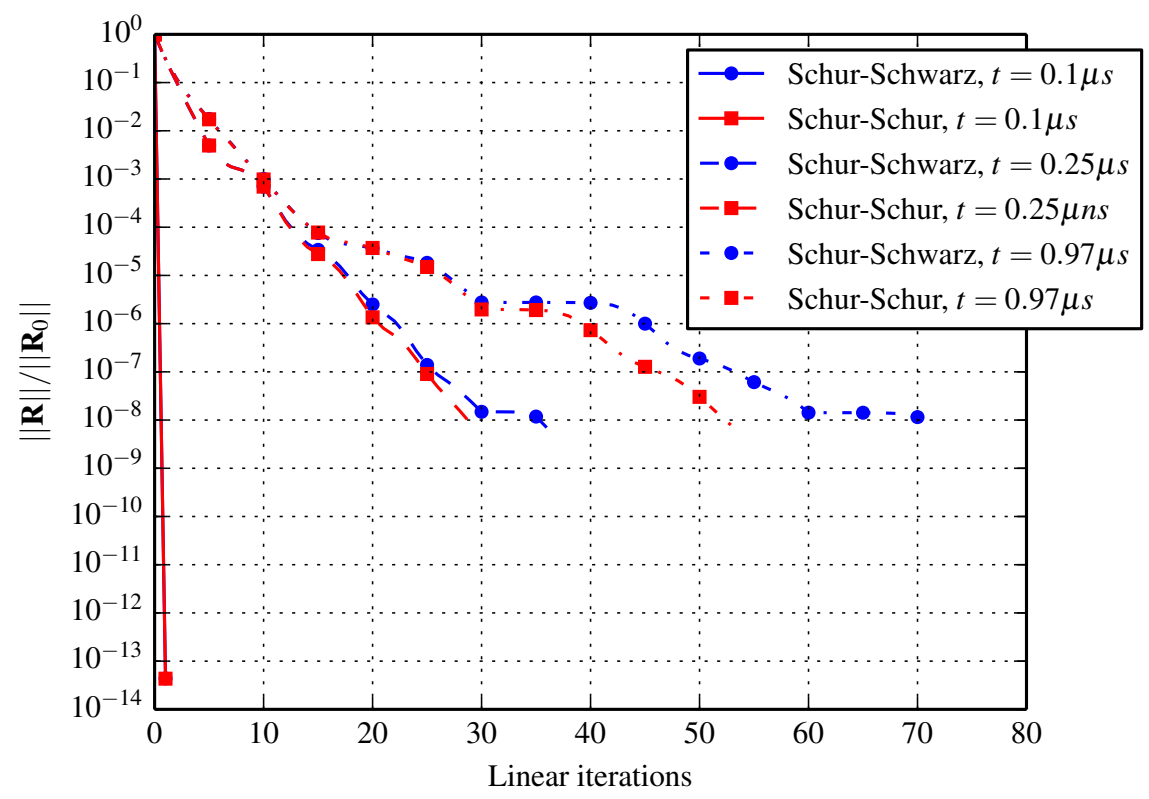

(b) Convergence behavior of the outer GMRES solver in the linear elastic regime, at the onset of plasticity and in the softening regime at times marked on Figure $9 a$ by orange vertical lines. 


\begin{tabular}{|c|c|c|c|c|c|c|c|c|c|}
\hline & \multicolumn{4}{|c|}{ LU solver } & \multicolumn{4}{|c|}{ Schur-ILU } \\
\hline & & $10^{2}$ & $20^{2}$ & $30^{2}$ & $40^{2}$ & $10^{2}$ & $20^{2}$ & $30^{2}$ & $40^{2}$ \\
\hline & $\mathrm{P} 1$ & 10.49 & 41.36 & 94.48 & 168.78 & 14.33 & 69.20 & 254.48 & 600.08 \\
\hline & $\mathrm{P} 2$ & 33.71 & 138.28 & 318.88 & 580.70 & 45.06 & 268.21 & 792.97 & 1741.09 \\
\hline â & P3 & 101.62 & 415.23 & 960.68 & 1734.03 & 147.30 & 703.06 & 1909.84 & 4081.25 \\
\hline$z$ & $\mathrm{P} 4$ & 260.49 & 1088.46 & 2425.82 & 4446.31 & 365.17 & 1654.68 & 4328.16 & 8833.92 \\
\hline
\end{tabular}

\begin{tabular}{|c|c|c|c|c|c|c|c|c|c|}
\hline & \multicolumn{4}{|c|}{ Schur-Schwarz } & \multicolumn{4}{|c|}{ Schur-Schur } \\
\hline & & $10^{2}$ & $20^{2}$ & $30^{2}$ & $40^{2}$ & $10^{2}$ & $20^{2}$ & $30^{2}$ & $40^{2}$ \\
\hline & $\mathrm{P} 1$ & 12.68 & 49.56 & 122.44 & 231.05 & 13.99 & 51.74 & 122.99 & 226.02 \\
\hline 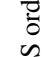 & $\mathrm{P} 2$ & 39.96 & 172.52 & 409.88 & 766.84 & 41.44 & 170.15 & 382.93 & 761.39 \\
\hline$\stackrel{\rho}{\mu}$ & P3 & 122.42 & 509.96 & 1177.34 & 2189.86 & 118.43 & 484.65 & 1153.38 & 2103.72 \\
\hline & $\mathrm{P} 4$ & 305.42 & 1241.48 & 2876.39 & 5273.26 & 291.62 & 1194.01 & 2765.66 & 5047.98 \\
\hline
\end{tabular}

Table 3: Overall computational time (in seconds) to simulate the shearband benchmark example as function of $h$ - and $k$-refinement. Note that LU direct solver performs better than all three preconditioners. 
First, strong scaling studies on 100 by 100 and 200 by 200 meshes are conducted. The problem is decomposed with increasingly smaller partitions but the number of cores per partition is kept fixed thus increasing the total number of cores used to solve the problem. Statistics of the partitions used for the 200 by 200 simulations are presented in Table 4. These particular partitions are based on optimized graph algorithms in METIS for our specific descretization.

\begin{tabular}{c|c|c|c|c}
\multirow{2}{*}{$\begin{array}{c}\text { number of } \\
\text { processors }\end{array}$} & \multicolumn{2}{|c|}{$\begin{array}{c}\text { number of equations } \\
\text { per partition }\end{array}$} & \multicolumn{2}{|c}{$\begin{array}{c}\text { number of ghost elements } \\
\text { per partition }\end{array}$} \\
& AVG & STD & AVG & STD \\
\hline 16 & 12525.0 & 96.1 & 98.8 & 62.3 \\
32 & 6262.5 & 93.9 & 79.0 & 53.3 \\
64 & 3131.2 & 48.3 & 59.3 & 32.2 \\
128 & 1565.6 & 26.3 & 42.2 & 23.4
\end{tabular}

Table 4: Meshes and partitions statistics for a 200 by 200 mesh, means are denoted AVG and standard deviations by STD.

In Table 5 the results for the 100 by 100 mesh for time $t=0$ to $t=1.2 \mu \mathrm{s}$ are summarized. It shows that the LU solver is initially faster when a small number of cores are used to solve the problem but as the number of cores and the number of compute nodes used for the simulation increases, the proposed preconditioners are proving more efficient in utilizing the resources available. In particular it can be seen that a GMRES solver preconditioned by the Schur-Schwarz preconditioner, gives similar performance as the LU direct solver on 32 cores, however on 64 cores it outperforms the other solvers and converges significantly faster. Note that the Schur-Schur preconditioner also shows good parallel scalability, although on this relatively small problem converges slower than the other methods. It is also noted that the parallelization leads to an increased number of GMRES iterations. This increase is smaller for the Schur-Schur preconditioner that has a better condition number and provides a more accurate description of the linear operator. The average number of linear iterations (per a nonlinear iteration) shows that for the Schur-Schwarz preconditioner an increase of 2 GMRES iterations per Newton iteration is expected when the number of processors is multiplied by 2 . For the Schur-Schur preconditioner an increase of 1 GMRES iteration per Newton iteration is expected when the number of processors is multiplied by 2 .

The CPU times and computational speedup [74] for these simulations are presented in Figure 10. It is observed that the computation time of the LU solver is 
always better than that of the GMRES solver preconditioned by the Schur-Schur preconditioner, the computational speedup achieved with the later is better as the number of processors increase.

\begin{tabular}{ccc|ccccccc}
\multicolumn{3}{c}{ Processors } & \multicolumn{2}{c}{ LU } & \multicolumn{3}{c}{ Schur-Schwarz } & \multicolumn{3}{c}{ Schur-Schur } \\
$-\mathrm{n}$ & $-\mathrm{p}$ & $-\mathrm{np}$ & $\mathrm{CPU}[\mathrm{s}]$ & $\mathrm{CPU}[\mathrm{s}]$ & Tot. Iter. & Avg. Iter. & CPU [s] & Tot. Iter. & Avg. Iter. \\
\hline 1 & 4 & 4 & 2670.87 & $3600+$ & N/A & N/A & $3600+$ & N/A & N/A \\
1 & 8 & 8 & 1809.87 & 2929.00 & 5110 & 75.1 & 2892.17 & 3504 & 51.5 \\
1 & 16 & 16 & 1378.88 & 1861.63 & 5268 & 77.5 & 2250.42 & 3591 & 52.8 \\
2 & 16 & 32 & 1159.95 & 1176.04 & 5385 & 79.2 & 1757.05 & 3699 & 54.4 \\
4 & 16 & 64 & 1058.16 & 890.48 & 5621 & 82.6 & 1361.88 & 3749 & 55.1
\end{tabular}

Table 5: 100 by 100 mesh, $-n$ is the number of compute nodes, $-\mathrm{p}$ is the number of cores per compute node and -np is the total number of cores used for these simulation. CPU[s] represents the simulation wall time in seconds, Tot. Iter. gives the total number of GMRES iterations and Avg. Iter. gives the corresponding average number of linear iterations per Newton step.

The same trends are also observed on the 200 by 200 problem that was run for 15 time steps due to limitations of the allocated compute time on MIRA. The CPU time required to solve the problem with the LU solver is almost always longer than that required by the GMRES solver preconditioned with the Schur-Schwarz and Schur-Schur preconditioners, as reported in Table 6. The speedups shown in Figure 11, are also greater with the proposed preconditioners, especially for the Schur-Schwarz preconditioner that requires fewer matrix multiplications and hence less message passings during the simulations. While the iteration matrix of the Schur-Schwarz preconditioner has a worse spectral radius, which means more GMRES iterations per linear solve as presented in Figure 9a, the computational savings achieved by the reduced communications are sufficient for an overall faster solver/preconditioner pair. The average number of linear iterations per Newton step is increasing more for the Schur-Schwarz preconditioner on the 200 by 200 mesh than on the 100 by 100 mesh with 3 more iterations instead of 2 when the number of processors is increased by a factor of 2. The Schur-Schur complement present the same strong scaling with an increase of 1 GMRES iteration on average per Newton step.

Finally the weak scaling of the GMRES solver preconditioned with the SchurSchwarz and Schur-Schur preconditioners is investigated using increasingly larger meshes but keeping the size of each partition close to constant, as reported in Table 7 and compared to the weak scaling of the LU solver. Figure 12 summarizes 

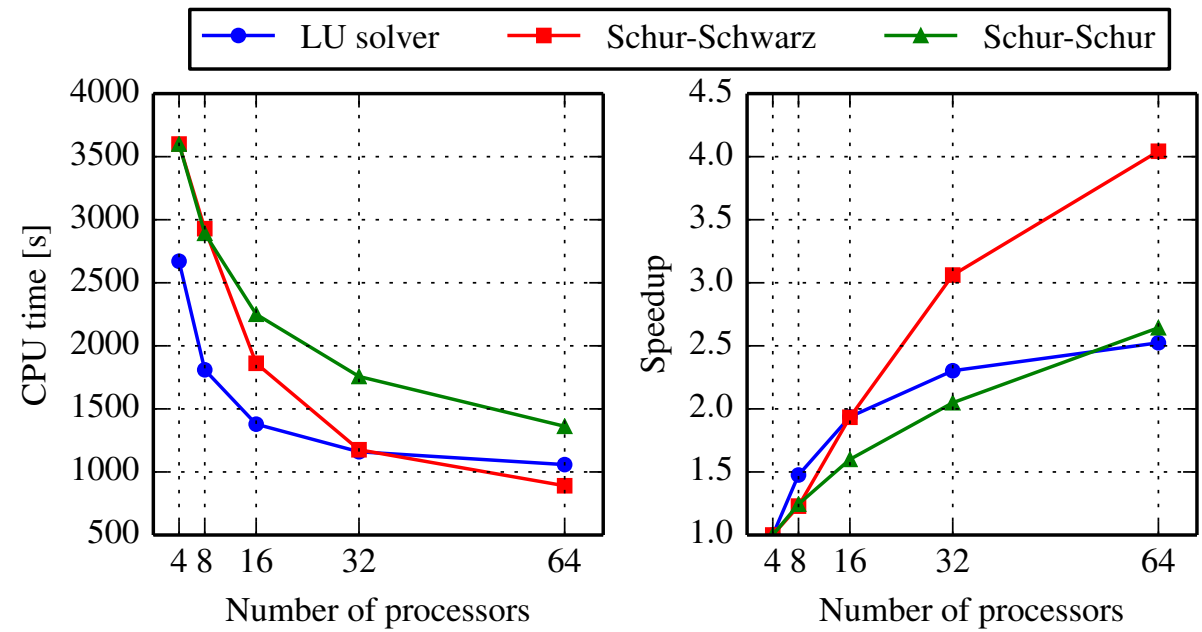

Figure 10: Strong scaling of the preconditioners compared to the LU solver for a 100 by 100 mesh. The Schur-Schwarz preconditioner outperforms the other methods as the number of processors increase.

\begin{tabular}{ccc|ccccccc}
\multicolumn{3}{c|}{ Processors } & \multicolumn{2}{c}{ LU } & \multicolumn{3}{c}{ Schur-Schwarz } & \multicolumn{3}{c}{ Schur-Schur } \\
-n & $-\mathrm{p}$ & $-\mathrm{np}$ & CPU [s] & CPU [s] & Tot Iter & Avg Iter & CPU [s] & Tot Iter & Avg Iter \\
\hline 2 & 8 & 16 & 2994.00 & 3342.02 & 2373 & 67.8 & 2906.31 & 1410 & 40.3 \\
4 & 8 & 32 & 2410.32 & 1985.20 & 2450 & 70.0 & 2024.08 & 1453 & 41.5 \\
8 & 8 & 64 & 2165.67 & 1335.78 & 2500 & 71.4 & 1533.18 & 1477 & 42.2 \\
16 & 8 & 128 & 2156.96 & 1029.80 & 2595 & 74.1 & 1291.75 & 1517 & 43.3
\end{tabular}

Table 6: 200 by 200 mesh, $-n$ is the number of compute nodes, $-\mathrm{p}$ is the number of cores per compute node and -np is the total number of cores used for these simulation. CPU[s] representes the simulation wall time in seconds, Tot. Iter. gives the total number of linear iterations and Avg. Iter. gives the average number of linear iterations for the simulation. 

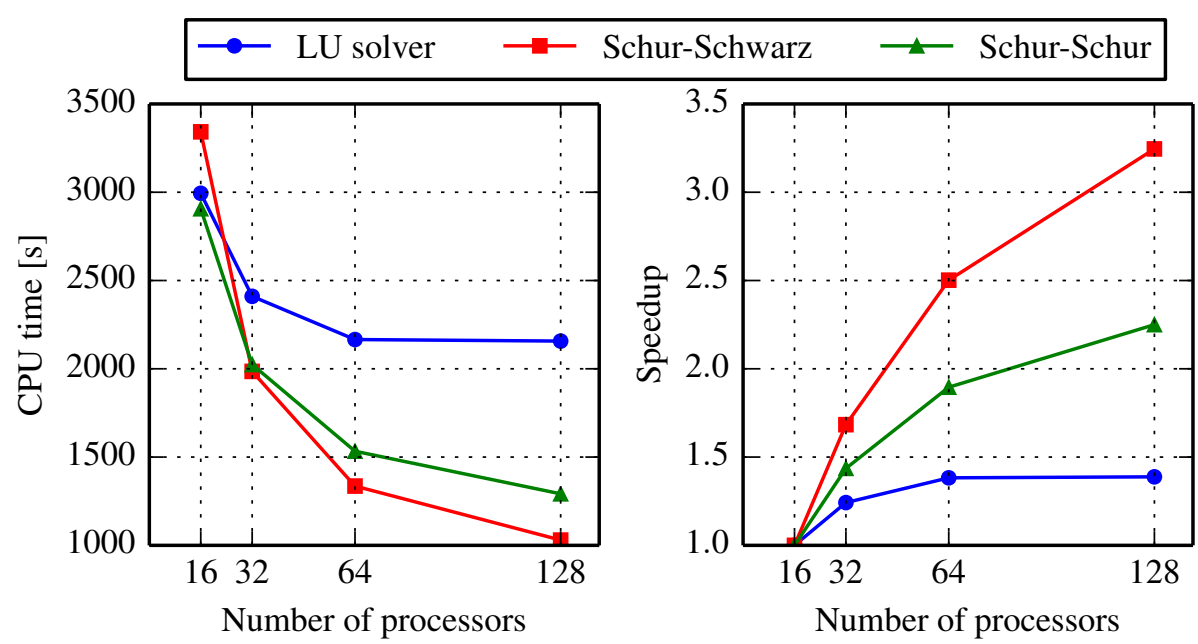

Figure 11: Strong scaling of the preconditioners compared to the LU solver for a 200 by 200 mesh.

the simulations CPU times and speedups. On small meshes the LU solver has comparable performances with the preconditioned GMRES methods but as the mesh size grows its computational time rapidly increasing whereas those of the GMRES preconditioned with the Schur-Schwarz and Schur-Schur preconditioners grows at lower rates. In contrast to the results obtained for the strong scaling, the weak scalability of the Schur-Schwarz preconditioner is not as good as that of the Schur-Schur preconditioner. For large scale problems the CPU time of the SchurSchur is eventually catching up with that of the Schur-Schwarz precondtioner as can be observed for the simulation ran on a 400 by 400 mesh.

\begin{tabular}{cccc} 
Mesh & Equations & Processors & Equations per processor \\
\hline 50 & 57,650 & 8 & 7206 \\
100 & 230,300 & 32 & 7196 \\
200 & 920,600 & 128 & 7192 \\
400 & $3,681,200$ & 512 & 7189
\end{tabular}

Table 7: Problem setting used for weak scaling studies. Choice of processors and mesh size that give approximately similar work per processor.

The scalability studies show that the Schur-Schwarz preconditioner has better strong scaling than the Schur-Schur preconditioner making it powerful on hard- 


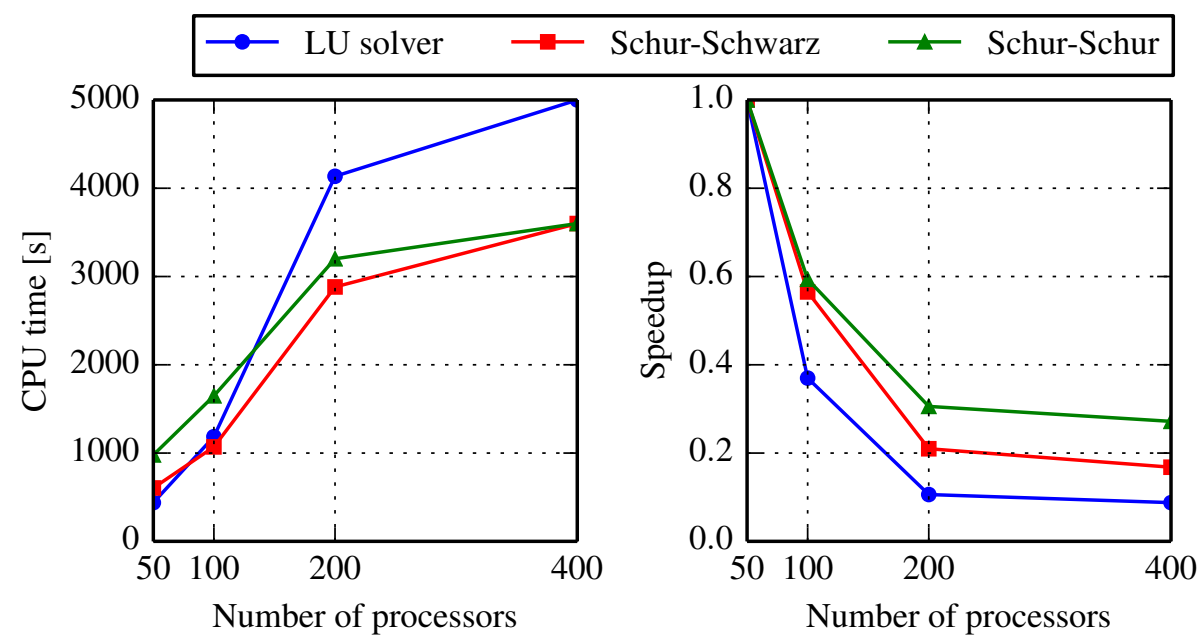

Figure 12: Weak scaling of the preconditioners compared to the LU solver

ware architecture using low amount of memory per processor but the Schur-Schur preconditioner exhibit better weak scaling abilities that makes it more efficient for very large systems of equations on massively parallel systems. This is further verified by the scaling study of both preconditioners in term of linear iteration as is presented in Table 8. It can be observed that the number of GMRES iterations stabilizes for the Schur-Schur complement whereas it continues to grow for the Schur-Schwarz preconditioner. This suggest that the Schur-Schur preconditioner is more suited for massively distributed systems.

\begin{tabular}{c|cccc}
\multirow{2}{*}{ Mesh } & \multicolumn{2}{|c}{ Schur-Schwarz } & \multicolumn{2}{c}{ Schur-Schur } \\
& Tot. Iter. & Avg. Iter. & Tot. Iter. & Avg. Iter. \\
\hline 50 & 981 & 24.9 & 959 & 27.4 \\
100 & 1853 & 52.9 & 1263 & 36.1 \\
200 & 2595 & 74.1 & 1517 & 43.3 \\
400 & 3231 & 92.3 & 1463 & 41.8
\end{tabular}

Table 8: Evolution of the number of linear iterations on increasingly large meshes 


\section{Conclusion}

Dynamic fracture of metals under high strain rates is an extremely difficult problem from the multiphysics perspective and the challenging numerics it requires. This so-called the shearband problem, can be described by a coupled thermo-mechanical set of PDEs with high nonlinearity levels, due to localized plasticity and heating, that increases with the formation and propagation of shearbands in the material. In order to resolve these shearbands accurately, which are on the orders of microns in width, very fine meshes, specialized basis functions (we consider assumed stress, high order Isogemetric based discretization) and parallel implementations are needed. This fine resolution of shearbands entails implicit schemes with very large Jacobian systems and a sparsity pattern that evolves with the deformation of shearbands, that needs to be solved at every time step.

To this end, our objective in this paper is to develop robust parallel preconsidioners to GMRES in order to alleviate the linear solve bottleneck of the analysis. The key idea is to partition the Jacobian into conservation laws and constitutive laws to exploit the localized block structure of constitutive laws and obtain elegantly a Schur complement of the system. The Schur complement is then approximated by a simpler expression for which a schwarz type method, so called SchurSchwarz preconditioner or a second inner Schur splitting, so called Schur-Schur preconditioner, are derived. A detailed presentation of the proposed preconditioners is provided with emphasis on the mathematical derivation, the block solvers employed and an eigenvalue analysis of the iteration matrix for each preconditioner.

Numerical experiments on a shearband benchmark problem are carried out in order to study the performance of preconditioners in comparison with a direct LU solver and an off-the-shelf GMRES preconditioned by ILU solver. First, we consider a serial implementation and study the behaviour of the solvers with the increase of the order of NURBS shape functions, so called k-refinement, as well as the standard h-refinement. As expected for all solvers, the solution time increases with both k- and h-refinements. On these relatively small systems, we find the direct LU solver to be the most efficient solver and converge the fastest while the GMRES preconditioned by ILU is clearly the worse. Nonetheless, the proposed two preconditioners perform also reasonably well and are closer to the LU solver. Finally, the solvers are implemented in parallel on a supercomputer at the Argonne National Lab. On these larger systems, a cross over is observed where both of the proposed preconditioners converge faster than the LU-direct solve. We conclude that the Schur-Schwarz preconditioner has better strong scaling that makes it more 
suitable for low memory per core architecture whereas the Schur-Schur preconditioner is more performant on isogeometric discretizations of higher degree and has better weak scaling making it suitable for very large simulation on massively parallel systems.

Future research directions include localization of some of the computation phases of the preconditioners as well as domain decomposition techniques in order to apply the preconditioners only to the region where shear bands are predominant.

\section{Acknowledgement}

This research used resources of the Argonne Leadership Computing Facility, which is a DOE Office of Science User Facility supported under Contract DEAC02-06CH11357. In addition, the financial support of the Department of Energy

through the Early Career Research Program, No. DE-SC0008 196, is gratefully acknowledged. 


\section{Appendix A. Multiplicative Schwarz method}

The multiplicative Schwarz method computes an approximate inverse of $\mathbf{A}$ that we will write $\mathbf{A}^{\#}$, using the successive inverse of $\mathbf{A}_{00}$ and $\mathbf{A}_{11}$. Hence this method requires both $\mathbf{A}_{00}$ and $\mathbf{A}_{11}$ to be invertible matrices. Using the following notations

$$
\mathbf{B}_{0}=\left[\begin{array}{cc}
\mathbf{A}_{00}^{-1} & \mathbf{0} \\
\mathbf{0} & \mathbf{1}
\end{array}\right] \quad \text { and } \quad \mathbf{B}_{1}=\left[\begin{array}{cc}
\mathbf{1} & \mathbf{0} \\
\mathbf{0} & \mathbf{A}_{11}^{-1}
\end{array}\right]
$$

one iteration of this method between steps $n$ and $n+1$ is derived as follows

$$
\begin{gathered}
\mathbf{r}^{n}=\mathbf{b}-\mathbf{A} \mathbf{u}^{n}, \\
\mathbf{u}^{n+1 / 2}=\mathbf{u}^{n}+\mathbf{B}_{0} \mathbf{r}^{n}, \\
\mathbf{r}^{n+1 / 2}=\mathbf{b}-\mathbf{A} \mathbf{u}^{n+1 / 2}, \\
\mathbf{u}^{n+1}=\mathbf{u}^{n+1 / 2}+\mathbf{B}_{1} \mathbf{r}^{n+1 / 2} .
\end{gathered}
$$

an approximate solution to (12) can be computed as

$$
\mathbf{u}=\left[\mathbf{B}_{0}+\mathbf{B}_{1}-\mathbf{B}_{1} \mathbf{A} \mathbf{B}_{0}\right] \mathbf{b}
$$

and the multiplicative Schwarz operator can be defined as

$$
\mathbf{A}^{\#}=\mathbf{B}_{0}+\mathbf{B}_{1}-\mathbf{B}_{1} \mathbf{A} \mathbf{B}_{0}
$$

which can be expanded to obtain the final expression

$$
\begin{aligned}
\mathbf{A}^{\#} & =\left[\begin{array}{cc}
\mathbf{A}_{00}^{-1} & \mathbf{0} \\
\mathbf{0} & \mathbf{1}
\end{array}\right]+\left[\begin{array}{cc}
\mathbf{1} & \mathbf{0} \\
\mathbf{0} & \mathbf{A}_{11}^{-1}
\end{array}\right]-\left[\begin{array}{cc}
\mathbf{1} & \mathbf{0} \\
\mathbf{0} & \mathbf{A}_{11}^{-1}
\end{array}\right]\left[\begin{array}{cc}
\mathbf{A}_{00} & \mathbf{A}_{01} \\
\mathbf{A}_{10} & \mathbf{A}_{11}
\end{array}\right]\left[\begin{array}{cc}
\mathbf{A}_{00}^{-1} & \mathbf{0} \\
\mathbf{0} & \mathbf{1}
\end{array}\right] \\
& =\left[\begin{array}{cc}
\mathbf{A}_{00}^{-1} & -\mathbf{A}_{01} \\
-\mathbf{A}_{11}^{-1} \mathbf{A}_{10} \mathbf{A}_{00}^{-1} & \mathbf{A}_{11}^{-1}
\end{array}\right]
\end{aligned}
$$

Remark 1: Usualy the Schwarz method is used in Richardson iterations to obtain a converged value for $\mathbf{u}$ and equation (A.6) is applied to a residual ( $\mathbf{r}=\mathbf{b}-\mathbf{A u}$ ) to compute an update $\delta \mathbf{u}$.

Remark 2: As mentioned above, $\mathbf{u}_{0}$ and $\mathbf{u}_{1}$ are complement of each other in $\mathbf{u}$, hence $\mathbf{A}_{00}$ and $\mathbf{A}_{11}$ are not overlapping, this allows us to compute the final expression of $\tilde{\mathbf{A}}$. In the case of overlapping matrices $\mathbf{A}_{00}$ and $\mathbf{A}_{11}$ the block matrix multiplications do not hold.

\section{Appendix B. Material properties}


Table B.9: Material parameters used in the example problems

\begin{tabular}{lccc}
\hline Physical quantity name & Name & Value & SI base unit \\
\hline Young's modulus & $E$ & 200E9 & $\mathrm{kg} \cdot \mathrm{m}^{-1} \cdot \mathrm{s}^{-2}$ \\
Poisson's ratio & $v$ & 0.3 & - \\
Mass density & $\rho$ & 7830 & $\mathrm{~kg} \cdot \mathrm{m}^{-3}$ \\
Specific heat & $c_{p}$ & 448 & $\mathrm{~m}^{2} \cdot \mathrm{s}^{-2} \cdot \mathrm{K}^{-1}$ \\
Thermal conductivity & $\kappa$ & 803.5 & $\mathrm{~kg} \cdot \mathrm{m} \cdot \mathrm{s}^{-3} \cdot \mathrm{K}$ \\
Thermal softening parameter & $\delta$ & 0.8 & - \\
Thermal softening parameter & $k$ & 500 & $\mathrm{~K}$ \\
Reference temperature & $T_{r e f}$ & 293 & $\mathrm{~K}$ \\
Taylor-Quinney Coefficient & $\chi$ & 0.9 & - \\
Yield stress & $\sigma_{r e f}$ & $2 \mathrm{E} 9$ & $\mathrm{~kg} \cdot \mathrm{m}^{-1} \cdot \mathrm{s}^{-2}$ \\
Yield strain & $\gamma_{r e f}$ & 0.01 & - \\
Reference strain rate & $\dot{\gamma}_{r e f}$ & 0.001 & $\mathrm{~s}^{-1}$ \\
Strain hardening exponent & $\mathrm{N}$ & 0.01 & - \\
Rate sensitivity parameter & $\mathrm{m}$ & 70 & - \\
\hline
\end{tabular}

\section{References}

[1] T.W. Wright. The Physics and Mathematics of Adiabatic Shear Bands. Cambridge Monographs on Mechanics. Cambridge University Press, Cambridge, 2002.

[2] A. Marchand and J. Duffy. An experimental study of the formation process of adiabatic shear bands in a structural steel. Journal of the Mechanics and Physics of Solids, 36(3):251 - 283, 1988.

[3] T.W. Wright and J.W. Walter. On stress collapse in adiabatic shear bands. Journal of the Mechanics and Physics of Solids, 35(6):701 - 720, 1987.

[4] K.-M. Cho, S. Lee, S.R. Nutt, and J. Duffy. Adiabatic shear band formation during dynamic torsional deformation of an hy-100 steel. Acta Metallurgica et Materialia, 41(3):923 - 932, 1993.

[5] A.-S Bonnet-Lebouvier, A Molinari, and P Lipinski. Analysis of the dynamic propagation of adiabatic shear bands. International Journal of Solids and Structures, 39(16):4249 - 4269, 2002. 
[6] T.G. Shawki and R.J. Clifton. Shear band formation in thermal viscoplastic materials. Mechanics of Materials, 8(1):13 - 43, 1989.

[7] F. Armero and J.C. Simo. A priori stability estimates and unconditionally stable product formula algorithms for nonlinear coupled thermoplasticity. International Journal of Plasticity, 9(6):749 - 782, 1993.

[8] J. Lemaitre and J.L. Chaboche. Mechanics of Solid Materials. Cambridge University Press, Cambridge, 2nd edition, 1994.

[9] T.W. Wright and J.W. Walter. The asymptotic structure of an adiabatic shear band in antiplane motion. Journal of the Mechanics and Physics of Solids, 44(1):77 - 97, 1996.

[10] M. Zhou, G. Ravichandran, and A.J. Rosakis. Dynamically propagating shear bands in impact-loaded prenotched plates-ii. numerical simulations. Journal of the Mechanics and Physics of Solids, 44(6):1007-1032, 1996.

[11] S. Li, W.-K. Liu, A.J. Rosakis, T. Belytschko, and W. Hao. Mesh-free galerkin simulations of dynamic shear band propagation and failure mode transition. International Journal of Solids and Structures, 39(5):1213 1240, 2002.

[12] A. Seitz, A. Popp, and W.A. Wall. A semi-smooth newton method for orthotropic plasticity and frictional contact at finite strains. Computer Methods in Applied Mechanics and Engineering, 285(0):228 - 254, 2015.

[13] D. Peirce, C.F. Shih, and A. Needleman. A tangent modulus method for rate dependent solids. Computers and Structures, 18(5):875-887, 1984.

[14] R.K. Abu Al-Rub and G.Z. Voyiadjis. A finite strain plastic-damage model for high velocity impact using combined viscosity and gradient localization limiters: Part i - theoretical formulation. International Journal of Damage Mechanics, 15(4):293-334, 2006.

[15] S. Li, W.-K. Liu, D. Qian, P.R. Guduru, and A.J. Rosakis. Dynamic shear band propagation and micro-structure of adiabatic shear band. Computer Methods in Applied Mechanics and Engineering, 191(1-2):73-92, 2001. Micromechanics of Brittle Materials and Stochastic Analysis of Mechanical Systems. 
[16] T. Belytschko, H.-Y. Chiang, and E. Plaskacz. High resolution twodimensional shear band computations: imperfections and mesh dependence. Computer Methods in Applied Mechanics and Engineering, 119(1-2):1-15, 1994.

[17] S. Li and W.K. Liu. Numerical simulations of strain localization in inelastic solids using mesh-free methods. International Journal for Numerical Methods in Engineering, 48(9):1285-1309, 2000.

[18] S. Li, W. Hao, and W.-K. Liu. Mesh-free simulations of shear banding in large deformation. International Journal of Solids and Structures, 37(4850):7185-7206, 2000.

[19] W.K. Liu and C. McVeigh. Predictive multiscale theory for design of heterogeneous materials. Computational Mechanics, 42(2):147-170, 2008.

[20] C. McVeigh and W.K. Liu. Multiresolution continuum modeling of microvoid assisted dynamic adiabatic shear band propagation. Journal of the Mechanics and Physics of Solids, 58(2):187 - 205, 2010.

[21] B. Loret and J.H. Prevost. Dynamic strain localization in elasto-(visco)plastic solids, part 1. general formulation and one-dimensional examples. Computer Methods in Applied Mechanics and Engineering, 83(3):247-273, 1990.

[22] E.C. Aifantis. On the role of gradients in the localization of deformation and fracture. International Journal of Engineering Science, 30(10):1279-1299, 1992.

[23] R.C. Batra and C.H. Kim. Effect of thermal conductivity on the initiation, growth and bandwidth of adiabatic shear bands. International Journal of Engineering Science, 29(8):949 - 960, 1991.

[24] A. Needleman. Material rate dependence and mesh sensitivity in localization problems. Computer Methods in Applied Mechanics and Engineering, 67(1):69-85, 1988.

[25] S.N. Medyanik, W.K. Liu, and S. Li. On criteria for dynamic adiabatic shear band propagation. Journal of the Mechanics and Physics of Solids, 55(7):1439 - 1461, 2007. 
[26] M. Zhou, A. Needleman, and R.J. Clifton. Finite element simulations of shear localization in plate impact. Journal of the Mechanics and Physics of Solids, 42(3):423 - 458, 1994.

[27] T.W. Wright and R.C. Batra. The initiation and growth of adiabatic shear bands. International Journal of Plasticity, 1(3):205 - 212, 1985.

[28] R.C. Batra. The initiation and growth of, and the interaction among, adiabatic shear bands in simple and dipolar materials. International Journal of Plasticity, 3(1):75 - 89, 1987.

[29] C. McAuliffe and H. Waisman. Mesh insensitive formulation for initiation and growth of shear bands using mixed finite elements. Computational Mechanics, 51(5):807-823, 2013.

[30] C. McAuliffe and H. Waisman. A Pian-Sumihara type element for modeling shear bands at finite deformation. Computational Mechanics, pages 1-16, 2013.

[31] L. Berger-Vergiat, C. McAuliffe, and H. Waisman. Isogeometric analysis of shear bands. Computational Mechanics, pages 1-19, 2014.

[32] J. Lyness and C. Moler. Numerical differentiation of analytic functions. SIAM Journal on Numerical Analysis, 4(2):202-210, 1967.

[33] W. Squire and G. Trapp. Using complex variables to estimate derivatives of real functions. SIAM Review, 40(1):110-112, 1998.

[34] A. Pérez-Foguet, A. Rodríguez-Ferran, and A. Huerta. Numerical differentiation for local and global tangent operators in computational plasticity. Computer Methods in Applied Mechanics and Engineering, 189(1):277296, 2000.

[35] C. Miehe. Numerical computation of algorithmic (consistent) tangent moduli in large-strain computational inelasticity. Computer Methods in Applied Mechanics and Engineering, 134(3-4):223-240, 1996.

[36] Y. Saad and M. Schultz. Gmres: A generalized minimal residual algorithm for solving nonsymmetric linear systems. SIAM Journal on Scientific and Statistical Computing, 7(3):856-869, 1986. 
[37] H. van der Vorst. Bi-cgstab: A fast and smoothly converging variant of bi-cg for the solution of nonsymmetric linear systems. SIAM Journal on Scientific and Statistical Computing, 13(2):631-644, 1992.

[38] R.S. Tuminaro and C. Tong. Parallel smoothed aggregation multigrid: Aggregation strategies on massively parallel machines. In Proceedings of the 2000 ACM/IEEE Conference on Supercomputing, Supercomputing '00, Washington, DC, USA, 2000. IEEE Computer Society.

[39] M.W. Gee, C.M. Siefert, J.J. Hu, R.S. Tuminaro, and M.G. Sala. ML 5.0 smoothed aggregation user's guide. Technical Report SAND2006-2649, Sandia National Laboratories, 2006.

[40] I. Ipsen. A note on preconditioning nonsymmetric matrices. SIAM Journal on Scientific Computing, 23(3):1050-1051, 2001.

[41] V. Howle, R. Kirby, and G. Dillon. Block preconditioners for coupled physics problems. SIAM Journal on Scientific Computing, 35(5):S368S385, 2013.

[42] H. Elman, V.E. Howle, J. Shadid, R. Shuttleworth, and R. Tuminaro. A taxonomy and comparison of parallel block multi-level preconditioners for the incompressible Navier-Stokes equations. Journal of Computational Physics, 227(3):1790 - 1808, 2008.

[43] E.C. Cyr, J.N. Shadid, and R.S. Tuminaro. Stabilization and scalable block preconditioning for the Navier-Stokes equations. Journal of Computational Physics, 231(2):345 - 363, 2012.

[44] S.C. Eisenstat and H.F. Walker. Choosing the forcing terms in an inexact newton method. SIAM Journal on Scientific Computing, 17(1):16-32, 1996.

[45] B.T. Aagaard, M.G. Knepley, and C.A. Williams. A domain decomposition approach to implementing fault slip in finite-element models of quasi-static and dynamic crustal deformation. Journal of Geophysical Research: Solid Earth, 118(6):3059-3079, 2013.

[46] S. Rhebergen, G. Wells, R. Katz, and A. Wathen. Analysis of block preconditioners for models of coupled magma/mantle dynamics. SIAM Journal on Scientific Computing, 36(4):A1960-A1977, 2014. 
[47] E.C. Cyr, J.N. Shadid, R.S. Tuminaro, R.P. Pawlowski, and L. Chacón. A new approximate block factorization preconditioner for two-dimensional incompressible (reduced) resistive mhd. SIAM Journal on Scientific Computing, 35(3):701-730, 2013.

[48] X.-C. Cai and M. Sarkis. A restricted additive schwarz preconditioner for general sparse linear systems. SIAM Journal on Scientific Computing, 21(2):792-797, 1999.

[49] T.J.R. Hughes, J.A. Cottrell, and Y. Bazilevs. Isogeometric analysis: Cad, finite elements, nurbs, exact geometry and mesh refinement. Computer Methods in Applied Mechanics and Engineering, 194(39-41):4135 - 4195, 2005.

[50] R. Bouclier, T. Elguedj, and A. Combescure. Locking free isogeometric formulations of curved thick beams. Computer Methods in Applied Mechanics and Engineering, 245-246(0):144 - 162, 2012.

[51] Y. Bazilevs, L. Beirao da Veiga, J.A. Cottrell, T.J.R. Hughes, and G. Sangalli. Isogeometric analysis: approximation, stability and error estimates for hrefined meshes. Mathematical Models and Methods in Applied Sciences, 16(07):1031-1090, 2006.

[52] J. Schur. Über Potenzreihen, die im Innern des Einheitskreises beschränkt sind. Journal für die reine und angewandte Mathematik, 147:205-232, 1917.

[53] M. Benzi and D.B. Szyld. Existence and uniqueness of splittings for stationary iterative methods with applications to alternating methods. Numerische Mathematik, 76(3):309-321, 1997.

[54] H. Elman, V.E. Howle, J. Shadid, R. Shuttleworth, and R. Tuminaro. Block preconditioners based on approximate commutators. SIAM Journal on Scientific Computing, 27(5):1651-1668, 2005.

[55] Y. Saad. A flexible inner-outer preconditioned gmres algorithm. SIAM Journal on Scientific Computing, 14(2):461-469, 1993.

[56] H.A. Schwarz. Über einen Grenzübergang durch alternierendes Verfahren. Vierteljahrsschrift der Naturforschenden Gesellschaft in Zürich, 15:272286, 1870. 
[57] C. McAuliffe and H. Waisman. A unified model for metal failure capturing shear banding and fracture. International Journal of Plasticity, 65(0):131$151,2015$.

[58] Argonne Leadership Computing Facility. Argonne leadership computing facility, Blue Gene/Q systems and supporting resources. Technical Report ALCF-BGQ-Resources-07152014, Argonne National Laboratory, 2014.

[59] J. Milano and P. Lembke. IBM System Blue Gene Solution: Blue Gene/Q Hardware Overview and Installation Planning. IBM Redbooks publication, 2013.

[60] G. Karypis and V. Kumar. A Fast and High Quality Multilevel Scheme for Partitioning Irregular Graphs. SIAM Journal on Scientific Computing, 20(1):359-392, January 1998.

[61] G. Karypis and V. Kumar. Parallel Multilevel series k-Way Partitioning Scheme for Irregular Graphs. SIAM Review, 41(2):278-300, January 1999.

[62] R.L. Taylor. FEAP - - A Finite Element Analysis Program, April 2011.

[63] S. Balay, J. Brown, K. Buschelman, V. Eijkhout, W. D. Gropp, D. Kaushik, M. G. Knepley, L. Curfman McInnes, B. F. Smith, and H. Zhang. PETSc users manual. Technical Report ANL-95/11 - Revision 3.4, Argonne National Laboratory, 2013.

[64] J. Brown, M.G. Knepley, D.A. May, L.C. McInnes, and B. Smith. Composable linear solvers for multiphysics. In Parallel and Distributed Computing (ISPDC), 2012 11th International Symposium on, pages 55-62, June 2012.

[65] B. Smith, L. Curfman McInnes, E. Constantinescu, M. Adams, S. Balay, J. Brown, M. Knepley, and H. Zhang. PETSc's software strategy for the design space of composable extreme-scale solvers. Preprint ANL/MCSP2059-0312, Argonne National Laboratory, 2012. DOE Exascale Research Conference, April 16-18, 2012, Portland, OR.

[66] P.R. Amestoy, I.S. Duff, and J.-Y. L'Excellent. Multifrontal parallel distributed symmetric and unsymmetric solvers. Computer Methods in Applied Mechanics and Engineering, 184(2-4):501 - 520, 2000. 
[67] R.D. Falgout, J.E. Jones, and U.M. Yang. The design and implementation of hypre, a library of parallel high performance preconditioners. In A. Bruaset and A. Tveito, editors, Numerical Solution of Partial Differential Equations on Parallel Computers, volume 51 of Lecture Notes in Computational Science and Engineering, pages 267-294. Springer Berlin Heidelberg, 2006.

[68] A. Henderson. ParaView Guide, A Parallel Visualization Application. Kitware Inc., 2008.

[69] J. D. Hunter. Matplotlib: A 2d graphics environment. Computing In Science \& Engineering, 9(3):90-95, 2007.

[70] B. Hendrickson and E. Rothberg. Improving the run time and quality of nested dissection ordering. SIAM Journal on Scientific Computing, 20(2):468-489, 1998.

[71] F. Pellegrini, J. Roman, and P.R. Amestoy. Hybridizing nested dissection and halo approximate minimum degree for efficient sparse matrix ordering. Concurrency: Practice and Experience, 12(2-3):69-84, 2000.

[72] A. George and J.W.H. Liu. A fast implementation of the minimum degree algorithm using quotient graphs. ACM Transactions on Mathematical Software, 6(3):337-358, September 1980.

[73] P.R. Amestoy, T.A. Davis, and I.S. Duff. Algorithm 837: Amd, an approximate minimum degree ordering algorithm. ACM Trans. Math. Softw., 30(3):381-388, September 2004.

[74] D.J. Kuck, Y. Muraoka, and S.-C. Chen. On the number of operations simultaneously executable in fortran-like programs and their resulting speedup. Computers, IEEE Transactions on, C-21(12):1293-1310, Dec 1972. 